\title{
A Semi-Analytical Model for the Simulation of the Isolation of the Vibration Due to a Harmonic Load Using Pile Rows Embedded in a Saturated Half-Space
}

\author{
Bin $\mathrm{Xu}^{1,2}$, Jian-Fei $\mathrm{Lu}^{3, *}$ and Jian-Hua Wang ${ }^{4}$ \\ ${ }^{I}$ Department of Civil Engineering, Shanghai JiaoTong University, Shanghai, 200030, P.R. China \\ ${ }^{2}$ Department of Civil Engineering, Nanchang Institute of Technology, Nanchang, Jianxi, 330099, P.R. China \\ ${ }^{3}$ Department of Civil Engineering, Jiangsu University, Zhenjiang, Jiangsu, 212013, P.R. China \\ ${ }^{4}$ Department of Civil Engineering, Shanghai Jiao Tong University, Shanghai 200030, P.R. China
}

\begin{abstract}
The isolation of the vibration due to a harmonic vertical load using pile rows embedded in a saturated poroelastic half-space is investigated in this study. Using the fundamental solution for a circular patch load and Muki's method, the second kind of Fredholm integral equations describing the dynamical interaction between the pile rows and the saturated poroelastic half-space are obtained. Numerical solution of the integral equations yields the dynamic response of the pile-half-space system. The vibration isolation effect of the pile rows is investigated via the proposed semi-analytical model. Numerical results indicate that stiffer piles have better isolation vibration effect than flexible piles. Moreover, the pile length and the spacing between neighboring piles in one pile row have significant influence on the isolation vibration effect of pile rows, while the influence of the spacing between neighboring pile rows is relatively smaller.
\end{abstract}

Keywords: Poroelastic half-space, Biot's theory, Fredholm integral equation, Pile rows, Passive vibration isolation.

\section{INTRODUCTION}

The vibration isolation methods can be classified into two categories: the active vibration isolation method and the passive isolation method. The active isolation method often is aimed to isolate the vibration source from its surrounding medium. Thus, the active vibration isolation facility is usually installed around the vibration source or at a very close distance from the source. The passive isolation facility, on the other hand, usually is far away from the source and surrounds the protected structure.

Roughly, there are two passive vibration isolation approaches: the trench (open or in-filled) approach and the pile approach (pile rows or sheet piles). To date, many researches concerning vibration isolation by trenches or piles have been conducted. For example, Emad and Manolis [1] utilized the boundary element method (BEM) with constant elements to research the vibration reduction effect of rectangular and circular open trenches. Beskos et al. [2] employed BEM with constant element to discuss the vibration reduction effects of the open and in-filled trench. Dasgupta et al. [3] applied the 3-D frequency domain BEM with the full space fundamental solution to investigate the isolation of the vibration due to a rigid surface foundation subjected to a harmonic loading via open and in-filled trenches. Avilles and Sanchez-Sesma [4]

*Address correspondence to this author at the Department of Civil Engineering, Jiangsu University, Zhenjiang, Jiangsu, 212013, P.R. China; Tel: +86(511)88791011; E-mail: 1jfdoctor@yahoo.com developed a theoretical model to study the vibration isolation effect of a pile row with the incident SV and Rayleigh waves as the sources. Kattis et al. [5, 6] used the 3-D frequency domain BEM to calculate the vibration isolation effect of a pile row under the same assumption. Recently, by means of the frequency domain BEM, the vibration isolation effect of four types of circular piles was studied by Tsai [7].

It should be noticed that many previous researches concerning vibration isolation treat the half-space as a singlephase elastic medium. Nevertheless, it is well-known that for the saturated soil, the pore fluid plays a very important role in the liquefaction and the shear failure of the soil. Thus, it is inappropriate to treat the saturated soil as a single-phase elastic medium. Although there have been many researches on the dynamic response of piles embedded in a poroelastic half-space, most of them are limited to the case of piles subjected to top harmonic loads. For example, based on Biot's theory [8-10], Zeng and Rajapakse [11] analyzed the steadystate dynamic response of an axially loaded pile embedded in a poroelastic half-space. Wang et al. [12] extended the problem to the dynamic response of pile groups embedded in a poroelastic half-space. Jin et al. [13] studied the timeharmonic response of a pile under lateral loadings embedded in a poroelastic half-space. Besides, the frequency domain dynamic response of a pile embedded in a half-space porous medium and subjected to $\mathrm{P}$ and $\mathrm{SV}$ waves was investigated by Lu \& Jeng [14]. However, up to now, very little attention has been paid on the vibration isolation using pile rows 
embedded in poroelastic soils. Based on Biot's theory, the effect of a pile row on the displacement amplitude reduction of soil subjected to an incident $\mathrm{S}$ wave was investigated in [15]. Also, using the Fourier-Bessel series expansion method with the aid of the translational addition theorem, the dynamic response of the poroelastic medium behind a pile row when subjected to incident elastic waves was studied by Cai et al. [16]. Based on Biot's theory and integral equation method, numerical simulations of the isolation of the moving loading induced vibration by pile rows embedded in a homogeneous or layered poroelastic half-space were conducted in $[17,18]$, respectively.

In this paper, based on Muki's method for piles [19, 20] and the fundamental solution for a circular patch load, a semi-analytical model for analyzing the isolation of the vibration due to a fixed vertical harmonic load using pile rows is developed. Compared with the researches in $[17,18]$, the most important contribution of this paper is that both the influence of the vertical and horizontal deformation of pile rows on the isolation effect is considered. As a result, three integral equations describing the vertical and horizontal pilehalf-space interaction are needed for each pile. The remainder of the paper is organized as follows. In the second Section, Biot's theory is outlined. In the third Section, the approach for establishing the fundamental solution for a vertical and a horizontal circular patch load applied in the poroelastic half-space is sketched. In the fourth Section, the Fredholm integral equations describing the vertical and horizontal interaction between the piles and poroelastic half-space are established. In the fifth Section, the amplitude reduction ratio assessing the isolation vibration effect is defined. In the sixth Section, some numerical examples and corresponding analysis are presented. Finally, in the seventh Section, based on the research in this study, some conclusions are drawn.

\section{BIOT'S THEORY}

In this study, the soil is considered as the porous medium, which is described by Biot's theory [8-10]. Equations of motion for the bulk material and the pore fluid are expressed in terms of the solid displacement $\left(u_{i}\right)$ and the infiltration displacement $\left(w_{i}\right)$ as follows [8-10]

$$
\begin{aligned}
& \mu u_{i, j j}+\left(\lambda+\alpha^{2} M+\mu\right) u_{j, j i}+\alpha M w_{j, j i}=\rho_{b} \ddot{u}_{i}+\rho_{f} \ddot{w}_{i} \\
& \alpha M u_{j, j i}+M w_{j, j i}=\rho_{f} \ddot{u}_{i}+m \ddot{w}_{i}+b_{p} \dot{w}_{i}
\end{aligned}
$$

where $\lambda$ and $\mu$ are Lame constants of the solid skeleton; $\rho_{b}$ is the bulk density of the porous medium, which is defined by $\rho_{b}=(1-\phi) \rho_{s}+\phi \rho_{f}$, where $\rho_{s}$ is the density of the solid skeleton, $\phi$ is the porosity of the porous medium and $\rho_{f}$ is the density of the pore fluid; $m=a_{\infty} \rho_{f} / \phi$ and $a_{\infty}$ is the tortuosity of the porous medium; $b_{p}=\eta / k, \eta$ and $k$ represent the viscosity of the pore fluid and the permeability of the porous medium, respectively; the superimposed dot on a variable denotes the time derivative.
Based on Biot's theory, the constitutive relation for a homogeneous porous medium has the following form [810]

$$
\begin{aligned}
& \sigma_{i j}=2 \mu \varepsilon_{i j}+\lambda \delta_{i j} e-\alpha \delta_{i j} p_{f} \\
& p_{f}=-\alpha M e+M \vartheta
\end{aligned}
$$

where $\sigma_{i j}$ is the total stress of bulk material; $\varepsilon_{i j}$ denotes the strain tensor of the solid skeleton; $p_{f}$ is the excess pore fluid pressure and $\delta_{i j}$ is the Kronecker delta; $e$ and $\vartheta$ are the dilatation of the solid skeleton and the fluid volume increment for a unit volume porous medium.

To derive the general solutions for Biot's equations, the Fourier transform with respect to time and frequency is involved [21], which is defined as follows

$$
\begin{aligned}
& \bar{f}(\omega)=\int_{-\infty}^{+\infty} f(t) e^{-i \omega t} d t \\
& f(t)=\frac{1}{2 \pi} \int_{-\infty}^{+\infty} \bar{f}(\omega) e^{i \omega t} d \omega
\end{aligned}
$$

where $f(t)$ represents a function in the time domain, $\bar{f}(\omega)$ is the Fourier transform of $f(t), t$ and $\omega$ denote time and frequency, respectively, and a bar over a variable denotes the Fourier transform.

Based on the Helmholtz decomposition method, the solid displacement in the frequency domain has the following decomposition

$\bar{u}_{i}=\bar{\varphi}_{f, i}+\bar{\varphi}_{s, i}+e_{i j k} \bar{\psi}_{k, j}$

where $\bar{\varphi}_{f}, \bar{\varphi}_{s}$ and $\bar{\psi}_{k}(k=1,2,3)$ are the scalar and vector potentials for the porous medium and $e_{i j k}$ is the Levi-Civita symbol. Moreover, in the Cartesian coordinate system, the vector potential $\bar{\psi}_{k}(k=1,2,3)$ satisfies the following condition

$\bar{\psi}_{i, i}=0$

According to the analysis of Bonnet [22], although two displacement vectors are used in Biot's theory, four independent variables exist in the porous medium. Consequently, the pore pressure has the following expression

$$
\bar{p}_{f}=A_{f} \bar{\varphi}_{f, i i}+A_{s} \bar{\varphi}_{s, i i}
$$

The above equations for Biot's theory can be reduced to the following Helmholtz equations [23]

$$
\begin{aligned}
& \nabla^{2} \bar{\varphi}_{f}+k_{f}^{2} \bar{\varphi}_{f}=0 \\
& \nabla^{2} \bar{\varphi}_{s}+k_{s}^{2} \bar{\varphi}_{s}=0 \\
& \nabla^{2} \bar{\varphi}+k_{t}^{2} \bar{\varphi}=0
\end{aligned}
$$


Note that the expressions for the two constants $A_{f}$, $A_{s}$ in (9) and the three complex wave numbers $\left(k_{f}, k_{s}\right.$ and $k_{t}$ ) can be found in [23].

Once the potentials for the porous medium $\left(\bar{\varphi}_{f}, \bar{\varphi}_{s}\right.$ and $\bar{\varphi})$ are determined, the frequency domain displacement and pore pressure are given by (7), (9), while the stress of the porous medium is determined by (3).

\section{THE FUNDAMENTAL SOLUTION FOR A POROELASTIC HALF-SPACE SUBJECTED TO A CIRCULAR PATCH LOAD IN THE FREQUENCY DOMAIN}

As shown in the previous section, the governing equations of Biot's theory can be reduced to three Helmholtz equations for two scalar potentials and one vector potential. Herein, based on the potential method, the fundamental solution for a poroelastic half-space subjected to a uniform patch load over a circular region with radius $R$ (Fig. 2) will be established. The resultant of the patch load is assumed to be unity. Since the half-space considered here is axisymmetric with respect to the axis $\mathrm{z}$ (Fig. 2), it is more convenient to consider the current problem in a cylindrical coordinate system $(r, \theta, z)$.

In the cylindrical coordinate system, the vector potential for the solid skeleton and the pore fluid displacements can be represented by two scalar potentials, $\bar{\chi}$ and $\bar{\eta}$ [23], which satisfy the condition (8) automatically. Thus, in the cylindrical coordinate system, the Helmholtz equations (10)-(12) are reduced to

$\left(\frac{\partial^{2}}{\partial r^{2}}+\frac{1}{r} \frac{\partial}{\partial r}+\frac{1}{r^{2}} \frac{\partial^{2}}{\partial \theta^{2}}+\frac{\partial^{2}}{\partial z^{2}}\right) \bar{\varphi}_{f}+k_{f}^{2} \bar{\varphi}_{f}=0$

$\left(\frac{\partial^{2}}{\partial r^{2}}+\frac{1}{r} \frac{\partial}{\partial r}+\frac{1}{r^{2}} \frac{\partial^{2}}{\partial \theta^{2}}+\frac{\partial^{2}}{\partial z^{2}}\right) \bar{\varphi}_{s}+k_{s}^{2} \bar{\varphi}_{s}=0$

$\left(\frac{\partial^{2}}{\partial r^{2}}+\frac{1}{r} \frac{\partial}{\partial r}+\frac{1}{r^{2}} \frac{\partial^{2}}{\partial \theta^{2}}+\frac{\partial^{2}}{\partial z^{2}}\right) \bar{\chi}+k_{t}^{2} \bar{\chi}=0$

$\left(\frac{\partial^{2}}{\partial r^{2}}+\frac{1}{r} \frac{\partial}{\partial r}+\frac{1}{r^{2}} \frac{\partial^{2}}{\partial \theta^{2}}+\frac{\partial^{2}}{\partial z^{2}}\right) \bar{\eta}+k_{t}^{2} \bar{\eta}=0$

The displacements for the solid frame can be expressed in terms of the above potentials in the cylindrical coordinate system $(r, \theta, z)$ as follows

$$
\begin{aligned}
& \bar{u}_{r}=\frac{\partial \bar{\varphi}_{f}}{\partial r}+\frac{\partial \bar{\varphi}_{s}}{\partial r}+\frac{1}{r} \frac{\partial \bar{\chi}}{\partial \theta}+\frac{\partial^{2} \bar{\eta}}{\partial r \partial z} \\
& \bar{u}_{\theta}=\frac{1}{r} \frac{\partial \bar{\varphi}_{f}}{\partial \theta}+\frac{1}{r} \frac{\partial \bar{\varphi}_{s}}{\partial \theta}-\frac{\partial \bar{\chi}}{\partial r}+\frac{1}{r} \frac{\partial^{2} \bar{\eta}}{\partial \theta \partial z} \\
& \bar{u}_{z}=\frac{\partial \bar{\varphi}_{f}}{\partial z}+\frac{\partial \bar{\varphi}_{s}}{\partial z}-\frac{1}{r} \frac{\partial}{\partial r}\left(r \frac{\partial \bar{\eta}}{\partial r}\right)-\frac{1}{r^{2}} \frac{\partial^{2} \bar{\eta}}{\partial \theta^{2}}
\end{aligned}
$$

For the poroelastic half-space subjected to a harmonic load, potentials $\bar{\varphi}_{f}, \bar{\varphi}_{s}, \bar{\eta}$ and displacements $\bar{u}_{r}, \bar{u}_{z}, \bar{w}_{r}$, $\bar{w}_{z}$ and stresses $\bar{\sigma}_{r r}, \bar{\sigma}_{\theta \theta}, \bar{\sigma}_{z z}, \bar{\sigma}_{r z}$ may be expanded into the series (20), while potentials $\bar{\chi}$ and displacements $\bar{u}_{\theta}$, $\bar{w}_{\theta}$ and stress $\bar{\sigma}_{z \theta}$ are expanded into the series (21) [24]

$f_{1}(r, \theta, z, \omega)=\sum_{m=0}^{\infty} f_{1 m}(r, z, \omega) \cos (m \theta)$

$f_{2}(r, \theta, z, \omega)=\sum_{m=0}^{\infty} f_{2 m}(r, z, \omega) \sin (m \theta)$

where subscript $m$ denotes the order of the term in the series, and $f_{1}, f_{2}$ represent the corresponding potentials, displacements, the pore pressure and the stresses, respectively.

To derive the fundamental solutions for the poroelastic half-space in the cylindrical coordinate system $(r, \theta, z)$, the Hankel integral transform is employed [24]. The $m$-th order Hankel transform is defined by

$$
\begin{aligned}
& \hat{f}^{(m)}(\xi)=\int_{0}^{+\infty} r f(r) J_{m}(\xi r) d r \\
& f(r)=\int_{0}^{+\infty} \xi \hat{f}^{(m)}(\xi) J_{m}(\xi r) d \zeta
\end{aligned}
$$

where $J_{m}(*)$ denotes the $m$-th order first kind of Bessel function and a caret above a variable denotes the Hankel transform, and $\xi$ denotes the horizontal wavenumber.

Substituting the $m$-th order component of the potentials $\bar{\varphi}_{f}, \bar{\varphi}_{s}, \bar{\eta}, \bar{\chi}$ in equation (20) and (21) into (13)-(16) and performing $m$-th order Hankel transform with respect to the radial coordinate $r$, the general solutions of the potential $\hat{\bar{\varphi}}_{f m}, \hat{\bar{\varphi}}_{s m}, \hat{\bar{\eta}}_{m}, \hat{\bar{\chi}}_{m}$ in the frequency-wavenumber domain are obtained as follows

$$
\begin{aligned}
& \hat{\bar{\varphi}}_{f m}^{(m)}(\xi, z, \omega)=A_{m}(\xi, \omega) e^{\delta_{f} z}+B_{m}(\xi, \omega) e^{-\delta_{f} z} \\
& \hat{\bar{\varphi}}_{s m}^{(m)}(\xi, z, \omega)=C_{m}(\xi, \omega) e^{\delta_{s} z}+D_{m}(\xi, \omega) e^{-\delta_{s} z} \\
& \hat{\bar{\chi}}_{m}^{(m)}(\xi, z, \omega)=E_{m}(\xi, \omega) e^{\delta_{t} z}+F_{m}(\xi, \omega) e^{-\delta_{t} z} \\
& \hat{\bar{\eta}}_{m}^{(m)}(\xi, z, \omega)=G_{m}(\xi, \omega) e^{\delta_{t} z}+H_{m}(\xi, \omega) e^{-\delta_{t} z}
\end{aligned}
$$

where the superscript $m$ denotes the $m$-th order Hankel transform, and $A_{m}(\xi, \omega) \sim H_{m}(\xi, \omega)$ are arbitrary constants to be determined by the boundary condition and the continuity condition at the patch load plane (Fig. 2). In (24)-(27), $\delta_{f}$, $\delta_{s}$ and $\delta_{t}$ are complex numbers associated with the vertical wavenumbers of the $\mathrm{P}_{1}$, the $\mathrm{P}_{2}$ and the $\mathrm{S}$ wave of the porous medium and 


$$
\delta_{f}=\sqrt{\xi^{2}-k_{f}^{2}}, \delta_{s}=\sqrt{\xi^{2}-k_{s}^{2}}, \delta_{t}=\sqrt{\xi^{2}-k_{t}^{2}}
$$

Note that the real part of $\delta_{\alpha}, \alpha=f, s, t$ in (28) should be always non-negative to guarantee the bounded condition at infinity.

As mentioned above, when derive the integral equations for the pile rows, the fundamental solutions of the poroelastic half-space subjected to a vertical and a horizontal patch load over a circular domain with the radius equal to that of the piles are needed. These kinds of fundamental solutions can be derived by means of the general solution (24)-(27) and the displacement-potential relation, the pore pressurepotential relation and the stress-potential relation, the details of which are available in [14].

\section{THE FREDHOLM INTEGRAL EQUATIONS FOR THE PILE- HALF-SPACE SYSTEM}

\subsection{The Piles and the Half-Space System}

As shown in Fig. (1), the vibration source is a harmonic vertical patch load with frequency $f(f=\omega / 2 \pi)$ and applied to a circular surface domain with the diameter $D$. The passive vibration isolation system is multiple pile rows embedded in a poroelastic half-space. The total number of the piles is $m$. Each circular pile has the same diameter $d(d=2 R)$, length $L$, modulus $E_{p}$ and the same density $\rho_{p}$. The spacing between two neighboring piles in one pile row is $s$ and the separation between two adjacent pile rows is $q$. The distance between the first pile row and the center of the vibration source is $d_{s}$ (Fig. 1).

Following Muki and Sternberg $[19,20]$ and Pak \& Jennings [25], the pile-soil is decomposed into two subproblems: an extended poroelastic half-space and multiple fictitious piles. The response of the poroelastic half-space is governed by Biot's theory, while the fictitious pile is described by a 1-D bar and beam vibration theory. For simplicity, the $i$-th pile is used to illustrate the establishment of the integral equations.

For the $i$-th fictitious pile, its Young's modulus and the density are expressed as $[19,20]$,

$$
E_{p^{*}}^{(i)}=E_{p}^{(i)}-E_{s}, \rho_{p^{*}}^{(i)}=\rho_{p}^{(i)}-\rho_{b}(i=1,2, \cdots \cdots, m)
$$

where $E_{p}^{(i)}$ and $E_{s}$ are Young's modulus for the $i$-th pile and the poroelastic half-space with $E_{s}=\mu(3 \lambda+2 \mu) /(\lambda+\mu)$; $\rho_{p}^{(i)}, \rho_{b}$ are the densities for the $i$-th pile and the poroelastic half-space; $E_{p^{*}}^{(i)}$ and $\rho_{p^{*}}^{(i)}$ are Young's modulus and the density for the $i$-th fictitious pile.

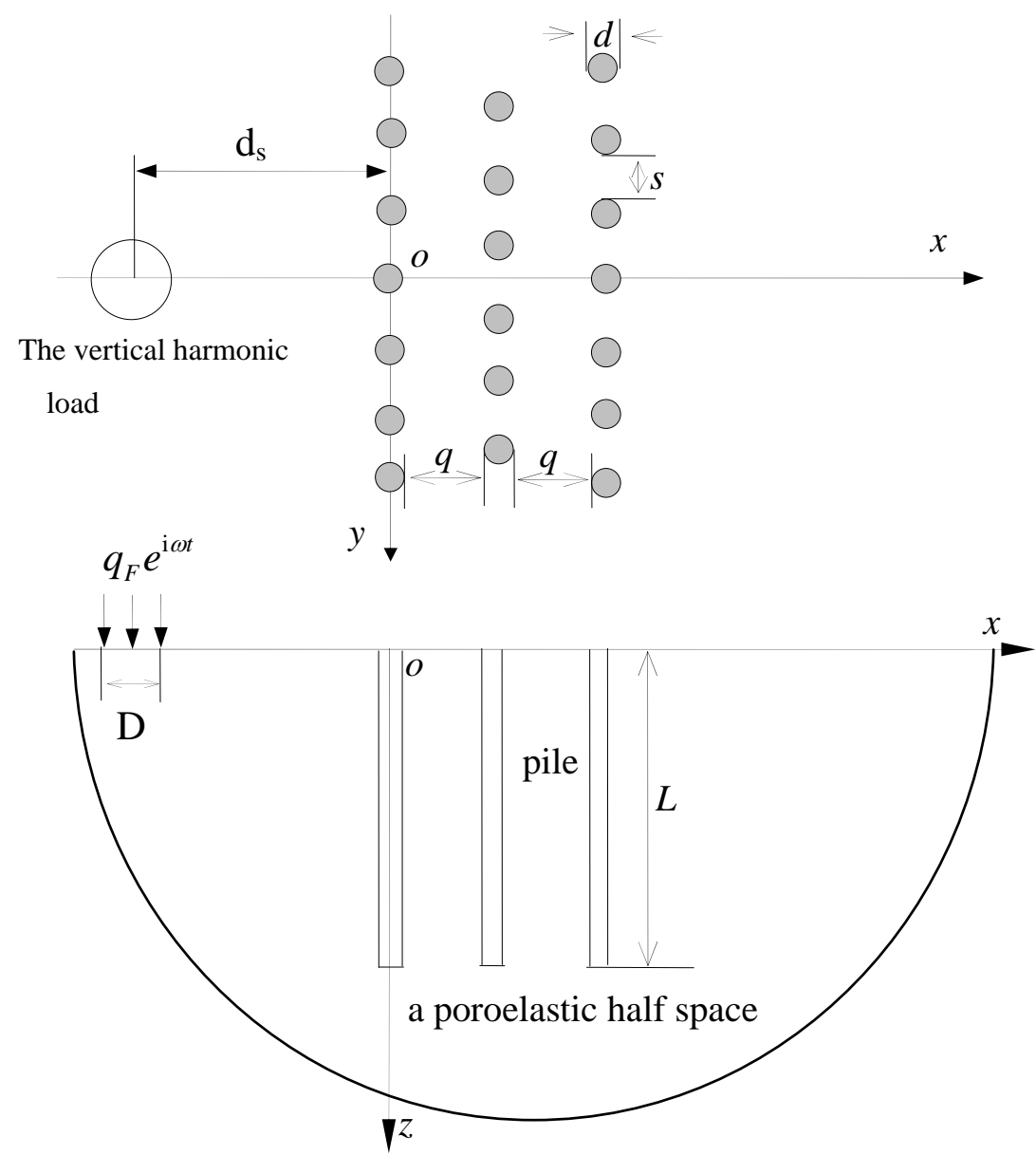

Fig. (1). Multiple pile rows used as vibration isolation system for a saturated poroelastic half space subjected to a harmonic vertical load. 
As shown in Fig. (3), it is assumed that the axial force, the shear force and the bending moment of the $i$-th fictitious pile are denoted by $\bar{N}_{*}^{(i)}(z), \bar{Q}_{k^{*}}^{(i)}(z)$ and $\bar{M}_{k^{*}}^{(i)}(z)$ $(k=x, y)$, respectively. The $i$-th fictitious pile is subjected to the vertical and the horizontal distributed loads $\bar{q}_{k}^{(i)}(z)$ $(k=x, y, z)$ along the length of the pile (Fig. 3). The top and the bottom of the $i$-th fictitious pile are subjected to vertical loads $\bar{N}_{*}^{(i)}(0), \bar{N}_{*}^{(i)}\left(L_{i}\right)$, horizontal loads $\bar{Q}_{k^{*}}^{(i)}(0)$, $\bar{Q}_{k^{*}}^{(i)}(L)(k=x, y)$ and bending moments $\bar{M}_{k^{*}}^{(i)}(0), \bar{M}_{k^{*}}^{(i)}(L)$ $(k=x, y)$, respectively.

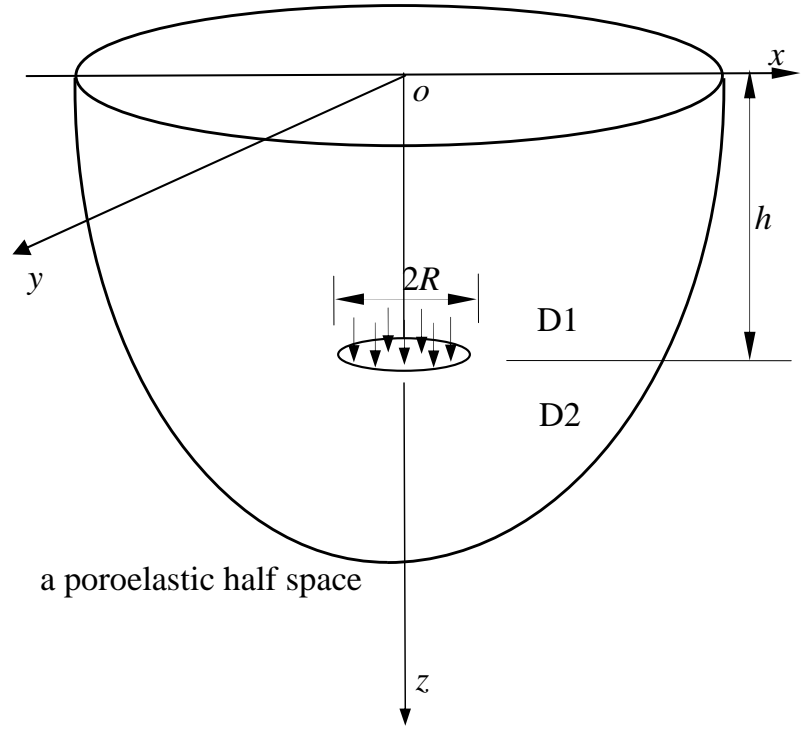

a
The extended poroelastic half-space are subjected to the following loads (Fig. 3): $\bar{q}_{k}^{(i)}(z)(k=x, y, z)$ which are uniformly distributed over the domain occupied by the $i$-th pile; $\bar{N}_{*}^{(i)}(0), \bar{Q}_{k^{*}}^{(i)}(0), \bar{M}_{k^{*}}^{(i)}(0) \quad(k=x, y)$ and $\bar{N}_{*}^{(i)}(L)$, $\bar{Q}_{k^{*}}^{(i)}(L), \bar{M}_{k^{*}}^{(i)}(L) \quad(k=x, y)$ which are applied over the circular domain $\Pi_{0}^{(i)}$ and $\Pi_{L}^{(i)}$, respectively.

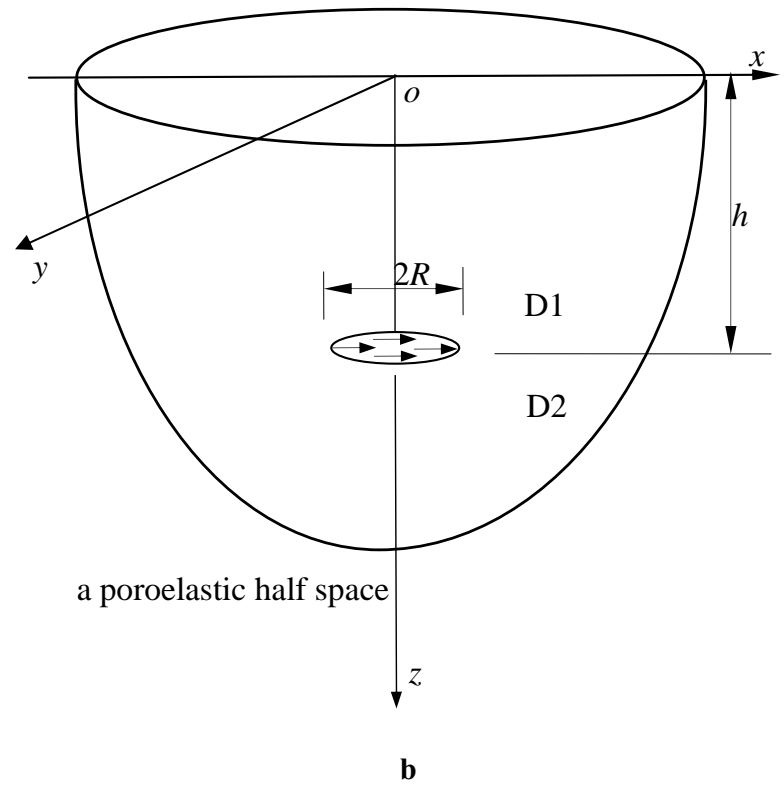

Fig. (2). A uniform circular patch load applied in a saturated poroelastic half space: (a) a vertical uniform circular patch load; (b) a horizontal uniform circular patch load.

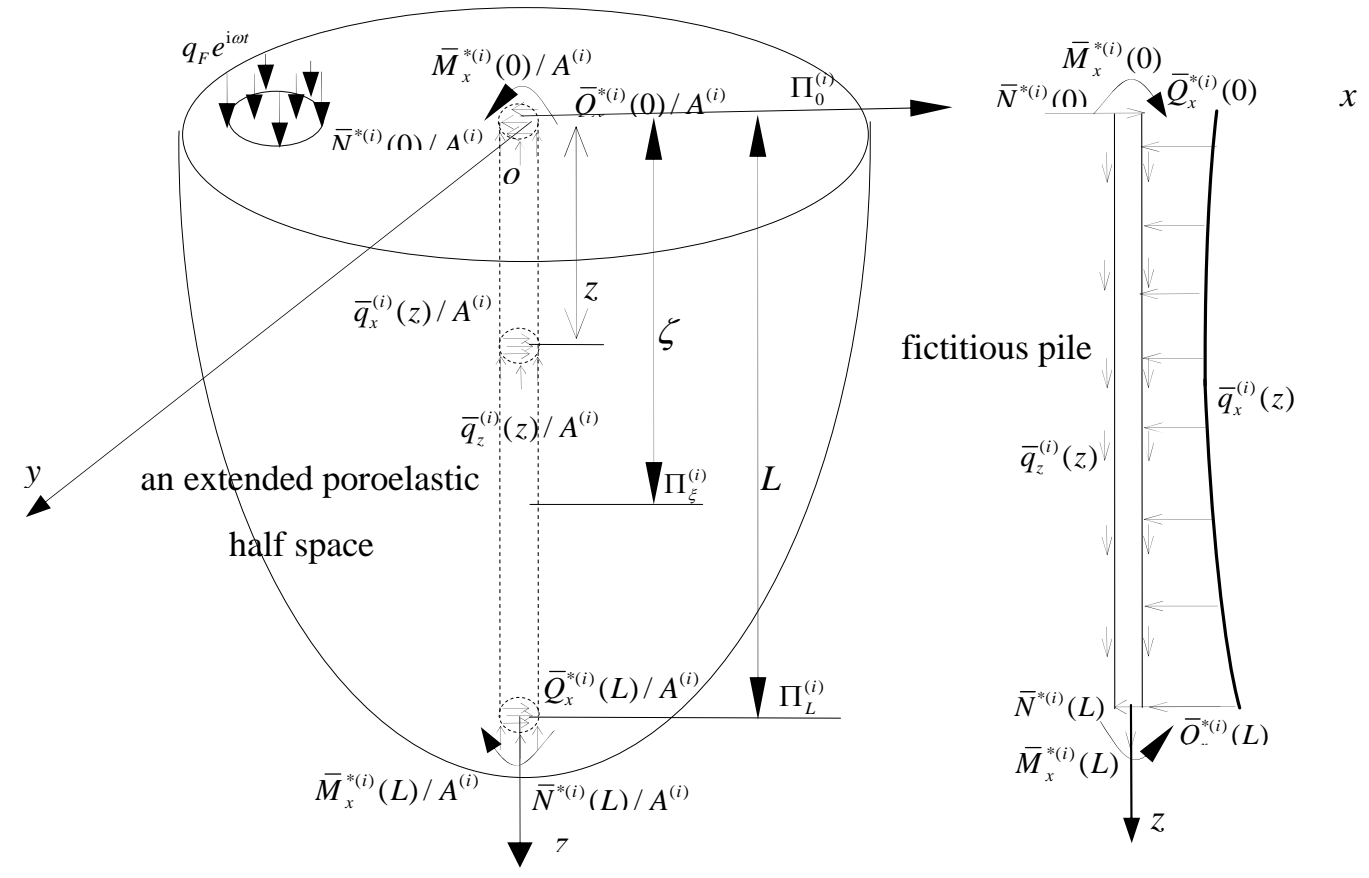

Fig. (3). The model for the $i$-th fictitious pile and the extended poroelastic half space. 
When the pile-half-space system is subjected to external loads, generally, the piles will undergo both vertical and horizontal deformation. As a result, the integral equations for the pile rows should account for the vertical and horizontal deformation of the piles. In this study, the compatibility condition that the axial strain of the fictitious piles and the vertical strain of the extended half-space along the piles' axis are equal is used to construct the integral equation accounting for the vertical coupling of the pile-half-space system. On the other hand, the condition that the rotary angles of the axis of the fictitious piles and those of the extended half-space along the axis of the piles are equal is used to establish the Fredholm integral equations accounting for the horizontal interaction within the system.

\subsection{The Equations of Motion for the Piles}

For the $i$-th fictitious pile, the vertical displacement $\bar{u}_{z^{*}}^{p(i)}(z)$, the distributed vertical load $\bar{q}_{z}^{(i)}(z)$ and the axial force $\bar{N}_{*}^{(i)}(z)$ satisfy the following relations

$$
\begin{aligned}
& \bar{q}_{z}^{(i)}(z)=-\frac{d \bar{N}_{*}^{(i)}(z)}{d z}-\rho_{p^{*}}^{(i)} A^{(i)} \omega^{2} \bar{u}_{z^{*}}^{(i)}(z) \\
& \bar{u}_{z^{*}}^{p(i)}(z)=\bar{u}_{z^{*}}^{(i)}(0)+\frac{1}{E_{p^{*}}^{(i)} A^{(i)}} \int_{0}^{z} \bar{N}_{*}^{(i)}(\eta) d \eta
\end{aligned}
$$

where $A^{(i)}$ denotes the cross-section area of the $i$-th pile.

The horizontal displacement $\bar{u}_{k^{*}}^{p(i)}(z)$, the distributed horizontal load $\bar{q}_{k}^{(i)}(z)$, the shear force $\bar{Q}_{k^{*}}^{(i)}(z)$ and the bending moment $\bar{M}_{k^{*}}^{(i)}(z) \quad(k=x, y)$ of the $i$-th fictitious pile satisfy the following relations

$\bar{q}_{k}^{(i)}(z)=-\frac{d \bar{Q}_{k^{*}}^{(i)}(z)}{d z}+\rho_{p^{*}}^{(i)} A^{(i)} \bar{u}_{k^{*}}^{p(i)}(z) \omega^{2},(k=x, y)$

$\frac{d \bar{M}_{k^{*}}^{(i)}(z)}{d z}=\bar{Q}_{k^{*}}^{(i)}(z),(k=x, y)$

Based on the beam theory, the bending moment $\left(\bar{M}_{k^{*}}^{(i)}(z), \quad(k=x, y)\right)$, the rotary angle of the pile $\left(\bar{\theta}_{k^{*}}^{p(i)}(z),(k=x, y)\right)$ and the horizontal displacement $\left(\bar{u}_{k^{*}}^{p(i)}(z),(k=x, y)\right)$ have the following form

$$
\begin{aligned}
& \bar{M}_{k^{*}}^{(i)}(z)=\int_{0}^{z} \bar{Q}_{k^{*}}^{(i)}(\zeta) d \zeta,(k=x, y) \\
& \bar{\theta}_{k^{*}}^{p(i)}(z)=\frac{1}{E_{p^{*}}^{(i)} I_{k}^{(i)}} \int_{0}^{z}(z-\zeta) \bar{Q}_{k^{*}}^{(i)}(\zeta) d \zeta+\bar{\theta}_{k^{*}}^{p(i)}(0) \\
& (k=x, y) \\
& \bar{u}_{k^{*}}^{(i)}(z)=\frac{1}{2 E_{p^{*}}^{(i)} I_{k}^{(i)}} \int_{0}^{z}(z-\zeta)^{2} \bar{Q}_{k^{*}}^{(i)}(\zeta) d \zeta+\bar{\theta}_{k^{*}}^{(i)}(0) z+\bar{u}_{k^{*}}^{(i)}(0), \\
& (k=x, y)
\end{aligned}
$$

where $I_{k}^{(i)}(k=x, y)$ is the second moment of the pile cross section.

\subsection{The Fredholm Integral Equations for the Vertical Interaction Between the Piles and the Half-Space}

As mentioned above, the vertical compatibility condition between the $i$-th pile and the extended half-space is fulfilled by requiring the axial strain of the $i$-th fictitious pile and the vertical strain of the extended half-space along the axis of the $i$-th pile to be equal

$\bar{\varepsilon}_{z^{*}}^{p(i)}(z)=\bar{\varepsilon}_{z}^{s(i)}(z), 0 \leq z \leq L \quad(i=1,2, \ldots \ldots, m)$

where $\bar{\varepsilon}_{z^{*}}^{p(i)}(z)$ represents the axial strain of the $i$-th fictitious pile, $\bar{\varepsilon}_{z}^{s(i)}(z)$ is the vertical strain of the extended half-space along the axis of the $i$-th pile.

The vertical strain of the extended half-space along the axis of the piles is composed of two parts: the first part is due to the free wave field, while the second part is due to the loads applied to the half-space by all the fictitious piles. Thus, the vertical strain of the extended half-space along the axis of the $i$-th pile can be written as

$$
\begin{aligned}
& \bar{\varepsilon}_{z}^{(i)}(z)=\bar{\varepsilon}_{z f}^{(i)}(z)+\sum_{j=1}^{m}\left[\bar{N}_{*}^{(j)}(0) \bar{\varepsilon}_{z z}^{(G)}\left(r_{i j}, z, 0\right)\right. \\
& \left.-\bar{N}_{*}^{(j)}(L) \bar{\varepsilon}_{z z}^{(G)}\left(r_{i j}, z, L\right)-\int_{0}^{L} \bar{q}_{z}^{(j)}(\zeta) \bar{\varepsilon}_{z z}^{(G)}\left(r_{i j}, z, \zeta\right) d \zeta\right] \\
& +\sum_{k=x, y}\left\{\sum _ { j = 1 } ^ { m } \left[-\bar{Q}_{k^{*}}^{(j)}(0) \bar{\varepsilon}_{k z}^{(G)}\left(r_{i j}, z, 0\right)+\bar{Q}_{k^{*}}^{(j)}\right.\right. \\
& \left.\left.(L) \bar{\varepsilon}_{k z}^{(G)}\left(r_{i j}, z, L\right)+\int_{0}^{L} \bar{q}_{k}^{(j)}(\zeta) \bar{\varepsilon}_{k z}^{(G)}\left(r_{i j}, z, \zeta\right) d \zeta\right]\right\}, \\
& (i=1,2, \ldots \ldots, m)
\end{aligned}
$$

where $\bar{\varepsilon}_{z f}^{(i)}(z)$ is the free field vertical strain due to the vertical harmonic source and $\bar{\varepsilon}_{z z}^{(G)}\left(r_{i j}, z, \zeta\right), \bar{\varepsilon}_{k z}^{(G)}\left(r_{i j}, z, \zeta\right)$ $(k=x, y)$ represents the vertical strain at the center of $\Pi_{z}^{(i)}$ due to a unit vertical and horizontal patch load at $\Pi_{\zeta}^{(j)}$, respectively (Fig. 3), $r_{i j}$ is the horizontal distance between the axis of the $i$-th and $j$-th pile. Note that if $i=j, r_{i j}$ will vanish.

Using (30)-(36) and (37), (38), the Fredholm integral equation describing the vertical interaction between the $i$-th pile and the extended half-space has the form

$$
\begin{aligned}
& \frac{\bar{N}_{*}^{(i)}(z)}{E_{p^{*}}^{(i)} A^{(i)}}+\bar{N}_{*}^{(i)}(z)\left[\bar{\varepsilon}_{z z}^{(G)}\left(r_{i i}, z, z^{+}\right)-\bar{\varepsilon}_{z z}^{(G)}\left(r_{i i}, z, z^{-}\right)\right] \\
& +\sum_{j=1}^{m}\left[\int_{0}^{L} \bar{N}_{*}^{(j)}(\zeta) \frac{\partial \bar{\varepsilon}_{z z}^{(G)}\left(r_{i j}, z, \zeta\right)}{\partial \zeta} d \zeta\right.
\end{aligned}
$$




$$
\begin{aligned}
& \left.-\int_{0}^{L_{j}} \bar{N}_{*}^{(j)}(\zeta) \bar{\chi}_{i j}^{(a)}(z, \zeta) d \zeta-\bar{u}_{z^{*}}^{p(j)}(0) \bar{\chi}_{i j}^{(b)}(z)\right] \\
& -\sum_{k=x, y}\left\{\sum _ { j = 1 } ^ { m } \left[\int _ { 0 } ^ { L } \left(\bar{Q}_{k^{*}}^{(j)}(\zeta) \frac{\partial \bar{\varepsilon}_{k z}^{(G)}\left(r_{i j}, z, \zeta\right)}{\partial z} d \zeta\right.\right.\right. \\
& +\int_{0}^{L} \bar{\kappa}_{i j}^{(a)}(\zeta) \bar{\varepsilon}_{k z}^{(G)}\left(r_{i j}, z, \zeta\right) d \zeta \\
& \left.\left.+\bar{\theta}_{k^{*}}^{p(j)}(0) \bar{\kappa}_{i j}^{(b)}(z)+\bar{u}_{k^{*}}^{p(j)}(0) \bar{\kappa}_{i j}^{(c)}(z)\right]\right\}=\bar{\varepsilon}_{z f}^{(i)}(z), \\
& (i=1,2, \ldots \ldots, m)
\end{aligned}
$$

where $\bar{\varepsilon}_{z z}^{(G)}\left(r_{i i}, z, z^{-}\right), \bar{\varepsilon}_{z z}^{(G)}\left(r_{i i}, z, z^{+}\right)$denote the vertical strain of the extended half-space at the center of $\Pi_{z}^{(i)}$ when the patch vertical load applied on $\Pi_{\xi}^{(i)}$ approaches $\Pi_{z}^{(i)}$ from up and down side, respectively, and

$$
\begin{aligned}
& \bar{\chi}_{i j}^{(a)}(z, \zeta)=\frac{\rho_{p^{*}}^{(j)} \omega^{2}}{E_{p^{*}}^{(j)}} \int_{\zeta}^{L} \bar{\varepsilon}_{z z}^{(G)}\left(r_{i j}, z, \eta\right) d \eta \\
& \bar{\chi}_{i j}^{(b)}(z)=\rho_{p^{*}}^{(j)} A^{(j)} \omega^{2} \int_{0}^{L} \bar{\varepsilon}_{z z}^{(G)}\left(r_{i j}, z, \eta\right) d \eta \\
& \bar{\kappa}_{i j}^{(a)}(\zeta)=\frac{\rho_{p^{*}}^{(j)} A \omega^{2}}{2 E_{p^{*}}^{(i)} I_{x}^{(i)}} \int_{0}^{L}(\zeta-\eta)^{2} \bar{Q}_{k^{*}}^{(i)}(\eta) d \eta \\
& \bar{\kappa}_{i j}^{(b)}(z)=\rho_{p^{*}}^{(j)} A^{(j)} \omega^{2} \int_{0}^{L} \zeta \bar{\zeta}_{k z}^{(G)}\left(r_{i j}, z, \zeta\right) d \zeta,(k=x, y) \\
& \bar{\kappa}_{i j}^{(c)}(z)=\rho_{p^{*}}^{(j)} A^{(j)} \omega^{2} \int_{0}^{L} \bar{\varepsilon}_{k z}^{(G)}\left(r_{i j}, z, \zeta\right) d \zeta,(k=x, y)
\end{aligned}
$$

Following the similar procedures, the surface vertical displacement $\bar{u}_{z s}\left(\mathbf{x}_{\perp}, z=0\right)$ for the poroelastic half space in the presence of pile rows can be calculated as follows

$$
\begin{aligned}
& \bar{u}_{z s}\left(\mathbf{x}_{\perp}, 0\right)=\bar{u}_{z f}\left(\mathbf{x}_{\perp}, 0\right)+\sum_{j=1}^{m}\left[-\int_{0}^{L} \bar{N}_{*}^{(j)}(\zeta) \frac{\partial \bar{U}_{z z}^{(G)}\left(r_{\mathbf{x}_{\perp} j}, 0, \zeta\right)}{\partial \zeta} d \zeta\right. \\
& \left.+\rho_{p^{*}}^{(j)} A^{(j)} \omega^{2} \int_{0}^{L} \bar{u}_{z^{*}}^{p(j)}(\zeta) \bar{U}_{z z}^{(G)}\left(r_{\mathbf{x}_{\perp} j}, 0, \zeta\right) d \zeta\right] \\
& +\sum_{k=x, y}\left\{\sum _ { j = 1 } ^ { m } \left[\int_{0}^{L_{j}} \bar{Q}_{k^{*}}^{(j)}(\zeta) \frac{\partial \bar{U}_{k z}^{(G)}\left(r_{\mathbf{x}_{\perp} j}, 0, \zeta\right)}{\partial z} d \zeta\right.\right. \\
& \left.\left.+\rho_{p^{*}}^{(j)} A^{(j)} \omega^{2} \int_{0}^{L_{j}} \bar{u}_{k^{\prime *}}^{p(j)}(\zeta) \bar{U}_{k z}^{(G)}\left(r_{\mathbf{x}_{\perp} j}, 0, \zeta\right) d \zeta\right]\right\}
\end{aligned}
$$

where $\bar{u}_{z f}\left(\mathbf{x}_{\perp}, 0\right)$ represents the free field vertical displacement, $\bar{U}_{z z}^{(G)}\left(r_{\mathbf{x}_{\perp} j}, 0, \zeta\right), \bar{U}_{k z}^{(G)}\left(r_{\mathbf{x}_{\perp} j}, 0, \zeta\right),(k=x, y)$ denotes the vertical displacement at the surface point $\mathbf{x}_{\perp}\left(\mathbf{x}_{\perp}=x \mathbf{i}+y \mathbf{j}\right)$ due to a unit patch vertical and horizontal load applied at $\Pi_{\xi}^{(j)}$, respectively, $r_{\mathbf{x}_{\perp} j}$ is the horizontal dis- tance between the surface point $\mathbf{x}_{\perp}$ and the axis of the $j$-th pile.

In equation (39), $\bar{u}_{z^{*}}^{(i)}(0)(i=1,2, \ldots \ldots, m)$ are also unknown. Thus, additional equations for $\bar{u}_{z^{*}}^{(i)}(0)$ $(i=1,2, \ldots \ldots, m)$ are needed for the closure of the system. The supplementary equations for $\bar{u}_{z^{*}}^{p(i)}(0)$ can be derived assuming that the vertical displacement of the $i$-th pile top and the vertical displacement of the extended half-space at the center of $\Pi_{0}^{(i)}$ are equal, i.e., $\bar{u}_{z^{*}}^{p(i)}(0)=\bar{u}_{z}^{s(i)}(0)$. Note that $\bar{u}_{z}^{s(i)}(0)$ can be obtained via (41) by setting $\mathbf{x}_{\perp}$ coincide with the center of the $i$-th pile top. Thus, using (30)-(36) and (41), the following supplementary equations for $\bar{u}_{z^{*}}^{p(i)}(0)$ are derived

$$
\begin{aligned}
& \sum_{j=1}^{m}\left[-\int_{0}^{L} \bar{N}_{*}^{(j)}(\zeta) \frac{\partial \bar{U}_{z z}^{(G)}\left(r_{i j}, 0, \zeta\right)}{\partial \zeta} d \zeta+\int_{0}^{L} \bar{N}_{*}^{(j)}(\zeta) \bar{\chi}_{i j}^{(c)}(0, \zeta) d \zeta\right] \\
& +\sum_{j=1}^{m} \bar{u}_{z^{*}}^{p(j)}(0)\left[\bar{\chi}_{i j}^{(d)}(0)-\delta_{i j}\right] \\
& +\sum_{k=x, y}\left\{\sum _ { j = 1 } ^ { m } \left[\int _ { 0 } ^ { L _ { j } } \left(\bar{Q}_{k^{*}}^{(j)}(\zeta) \frac{\partial \bar{U}_{k z}^{(G)}\left(r_{i j}, 0, \zeta\right)}{\partial z} d \zeta\right.\right.\right. \\
& +\int_{0}^{L} \bar{\kappa}_{i j}^{(a)}(\zeta) \bar{U}_{k z}^{(G)}\left(r_{i j}, 0, \zeta\right) d \zeta+\bar{\theta}_{k^{*}}^{p(j)} \\
& \left.\left.(0) \bar{\kappa}_{i j}^{(d)}(0)+\bar{u}_{k^{*}}^{p(j)}(0) \bar{\kappa}_{i j}^{(e)}(0)\right]\right\} \\
& =-\bar{u}_{z f}^{(i)}(0), i=1,2, \ldots \ldots, m
\end{aligned}
$$

where $\delta_{i j}$ is the Kronecker delta and

$$
\begin{aligned}
& \bar{\chi}_{i j}^{(c)}(z, \zeta)=\frac{\rho_{p^{*}}^{(j)} \omega^{2}}{E_{p^{*}}^{(j)}} \int_{\zeta}^{L} \bar{U}_{z z}^{(G)}\left(r_{i j}, z, \eta\right) d \eta \\
& \bar{\chi}_{i j}^{(d)}(z)=\rho_{p^{*}}^{(j)} A^{(j)} \omega^{2} \int_{0}^{L} \bar{U}_{z z}^{(G)}\left(r_{i j}, z, \eta\right) d \eta \\
& \bar{\kappa}_{i j}^{(d)}(z)=\rho_{p^{*}}^{(j)} A^{(j)} \omega^{2} \int_{0}^{L} \zeta \bar{U}_{k z}^{(G)}\left(r_{i j}, z, \zeta\right) d \zeta,(k=x, y) \\
& \bar{\kappa}_{i j}^{(e)}(z)=\rho_{p^{*}}^{(j)} A^{(j)} \omega^{2} \int_{0}^{L} \bar{U}_{k z}^{(G)}\left(r_{i j}, z, \zeta\right) d \zeta,(k=x, y)
\end{aligned}
$$

\subsection{The Fredholm Integral Equations Accounting for the Horizontal Interaction the Piles and the Half-Space}

The horizontal compatibility between the $i$-th pile and the extended half-space is realized by assuming the rotary angle of the $i$-th fictitious pile $\left(\bar{\theta}_{k^{*}}^{p(i)}(z) \quad(k=x, y)\right)$ and the rotary angle of the extended half-space along the axis of the pile $\left(\bar{\theta}_{k}^{s(i)}(z)(k=x, y)\right)$ to be equal 
$\bar{\theta}_{k}^{s(i)}(z)=\bar{\theta}_{k^{*}}^{p(i)}(z), 0<z<L, i=1,2, \ldots \ldots, m, k=x, y$

where $\bar{\theta}_{k}^{s(i)}(z)$ and $\bar{\theta}_{k^{*}}^{p(i)}(z)(k=x, y)$ denote the rotary angle of the extended half-space and the $i$-th fictitious pile in the $x o z$ and the yoz plane, respectively.

Likewise, the rotary angle of the extended half-space along the axis the $i$-th pile consists of two parts: the first part is the contribution from the free wave field, while the second part is due to the loads applied to the half-space by the fictitious piles (Fig. 3). As a result, taking proper account of the discontinuity of the integrand, the rotary angle of the extended poroelastic half-space along the axis the $i$-th pile has the form

$$
\begin{aligned}
& \bar{\theta}_{k}^{s(i)}(z)=\bar{\theta}_{k f}^{(i)}(z)+\bar{Q}_{k^{*}}^{(i)}(z)\left[\bar{\phi}_{k k}^{(G)}\left(r_{i i}, z, z^{+}\right)-\bar{\phi}_{k k}^{(G)}\left(r_{i i}, z, z^{-}\right)\right] \\
& +\sum_{k^{\prime}=x, y}\left\{\sum _ { j = 1 } ^ { m } \left[\int_{0}^{L} \bar{Q}_{k^{*}}^{(i)}(\zeta) \frac{\partial \bar{\phi}_{k^{\prime} k}^{(G)}\left(r_{i j}, z, \zeta\right)}{\partial \zeta} d \zeta\right.\right. \\
& \left.\left.+\rho_{p^{*}}^{(j)} A^{(j)} \omega^{2} \int_{0}^{L} \bar{u}_{k^{*}}^{p(j)}(\zeta) \bar{\phi}_{k^{\prime} k}^{(G)}\left(r_{i j}, z, \zeta\right) d \zeta\right]\right\} \\
& +\sum_{j=1}^{m(j \neq i)}\left[-\int_{0}^{L} \bar{N}_{*}^{(j)}(\zeta) \frac{\partial \bar{\phi}_{z k}^{(G)}\left(r_{i j}, z, \zeta\right)}{\partial \zeta} d \zeta\right. \\
& \left.+\rho_{p^{*}}^{(j)} A^{(j)} \omega^{2} \int_{0}^{L} \bar{u}_{z^{*}}^{p(j)}(\zeta) \bar{\phi}_{z k}^{(G)}\left(r_{i j}, z, \zeta\right) d \zeta\right], \\
& (i=1,2, \ldots \ldots, m, k=x, y)
\end{aligned}
$$

where $\bar{\theta}_{k f}^{(i)}(z)(k=x, y)$ is the free field rotary angle along the axis of the $i$-th pile due to the harmonic vertical load, $\bar{\phi}_{k^{\prime} k}^{(G)}\left(r_{i j}, z, \zeta\right), \bar{\phi}_{z k}^{(G)}\left(r_{i j}, z, \zeta\right) \quad\left(k, k^{\prime}=x, y\right)$ represents the rotary angle at the center of $\Pi^{(i)}$ due to a unit patch horizontal and vertical load applied on $\Pi_{\zeta}^{(j)}$, respectively; $\bar{\phi}_{k k}^{(G)}\left(r_{i i}, z, z^{-}\right), \bar{\phi}_{k k}^{(G)}\left(r_{i i}, z, z^{+}\right) \quad(k=x, y)$ denote the rotary angle at the center of $\Pi_{z}^{(i)}$ when the patch load applied on $\Pi_{\zeta}^{(i)}$ located at $z^{-}$and $z^{+}$, respectively.

Using equations (30)-(36) and (44), (45), the following second kind of Fredholm integral equations in the $x o z$ and the $y o z$ plane are obtained as follows

$$
\begin{aligned}
& \bar{Q}_{k^{*}}^{(i)}(z)\left[\bar{\phi}_{k k}^{(G)}\left(r_{i i}, z, z^{+}\right)-\bar{\phi}_{k k}^{(G)}\left(r_{i i}, z, z^{-}\right)\right] \\
& +\sum_{k^{\prime}=x, y}\left\{\sum _ { j = 1 } ^ { m } \left[\int_{0}^{L} \bar{Q}_{k^{\prime *}}^{(i)}(\zeta) \frac{\partial \bar{\phi}_{k^{\prime} k}^{(G)}\left(r_{i j}, z, \zeta\right)}{\partial \zeta} d \zeta\right.\right. \\
& -\frac{1}{E_{p^{*}}^{(j)} I_{k}^{(j)}} \int_{0}^{z}(z-\zeta) \bar{Q}_{k^{\prime *}}^{(i)}(\zeta) d \zeta+\int_{0}^{L} \bar{\kappa}_{i j}^{(a)}(\zeta) \bar{\phi}_{k^{\prime} k}^{(G)}\left(r_{i j}, z, \zeta\right) d \zeta \\
& \left.\left.+\bar{\theta}_{k^{\prime *}}^{p(j)}(0) \bar{\Gamma}_{i j}^{(a)}(z)+\bar{u}_{k^{\prime *}}^{p(j)}(0) \bar{\Gamma}_{i j}^{(b)(j)}(z)\right]\right\} \\
& +\sum_{j=1}^{m(j \neq i)}\left[\int_{0}^{L} \bar{N}_{*}^{(j)}(\zeta) \frac{\partial \bar{\phi}_{z k}^{(G)}\left(r_{i j}, z, \zeta\right)}{\partial \zeta} d \zeta\right.
\end{aligned}
$$

$$
\begin{aligned}
& \left.-\int_{0}^{L} \bar{N}_{*}^{(j)}(\zeta) \bar{\Lambda}_{i j}^{(a)}(\mathrm{z}, \zeta) d \zeta-\bar{u}_{z^{*}}^{p(j)}(0) \bar{\Lambda}_{i j}^{(b)}(z)\right] \\
& =-\bar{\theta}_{k}^{f(i)}(z),(k=x, y ; i=1,2, \ldots \ldots, m)
\end{aligned}
$$

where

$$
\begin{aligned}
& \bar{\Gamma}_{i j}^{(a)}(z)=\rho_{p^{*}}^{(i)} A^{(i)} \omega^{2} \int_{0}^{L} \zeta \bar{\phi}_{k^{\prime} k}^{(G)}\left(r_{i j}, z, \zeta\right) d \zeta-1,\left(k, k^{\prime}=x, y\right) \\
& \bar{\Gamma}_{i j}^{(b)}(z)=\rho_{p^{*}}^{(i)} A^{(i)} \omega^{2} \int_{0}^{L} \bar{\phi}_{k^{\prime} k}^{(G)}\left(r_{i j}, z, \zeta\right) d \zeta,\left(k, k^{\prime}=x, y\right) \\
& \bar{\Lambda}_{i j}^{(a)}(z, \zeta)=\frac{\rho_{p^{*}}^{(j)} \omega^{2}}{E_{p^{*}}^{(j)}} \int_{\zeta}^{L} \bar{\phi}_{z k}^{(G)}\left(r_{i j}, z, \eta\right) d \eta,(k=x, y) \\
& \bar{\Lambda}_{i j}^{(b)}(z)=\rho_{p^{*}}^{(j)} A^{(j)} \omega^{2} \int_{0}^{L} \bar{\phi}_{z k}^{(G)}\left(r_{i j}, z, \eta\right) d \eta,(k=x, y)
\end{aligned}
$$

For the extended half-space, the horizontal displacement at the surface point $\left(\mathbf{x}_{\perp}, 0\right)$ along $k(k=x, y)$ direction has the following expression

$$
\begin{aligned}
& \bar{u}_{k s}\left(\mathbf{x}_{\perp}, 0\right)=\bar{u}_{k f}\left(\mathbf{x}_{\perp}, 0\right) \\
& +\sum_{j=1}^{m(j \neq i)}\left[-\int_{0}^{L_{j}} \bar{N}_{*}^{(j)}(\zeta) \frac{\partial \bar{U}_{z k}^{(G)}\left(r_{\mathbf{x}_{\perp} j}, 0, \zeta\right)}{\partial \zeta} d \zeta\right. \\
& \left.+\rho_{p^{*}}^{(j)} A^{(j)} \omega^{2} \int_{0}^{L} \bar{u}_{z^{*}}^{p(j)}(\zeta) \bar{U}_{z k}^{(G)}\left(r_{\mathbf{x}_{\perp} j}, 0, \zeta\right) d \zeta\right] \\
& +\sum_{k^{\prime}=x, y}\left\{\sum _ { j = 1 } ^ { m } \left[\int_{0}^{L} \bar{Q}_{k^{* *}}^{(j)}(\zeta) \frac{\partial \bar{U}_{k^{\prime} k}^{(G)}\left(r_{\mathbf{x}_{\perp} j}, 0, \zeta\right)}{\partial \zeta} d \zeta\right.\right. \\
& \left.\left.+\rho_{p^{*}}^{(j)} A^{(j)} \omega^{2} \int_{0}^{L} \bar{u}_{k^{\prime *}}^{p(j)}(\zeta) \bar{U}_{k^{\prime} k}^{(G)}\left(r_{\mathbf{x}_{\perp} j}, 0, \zeta\right) d \zeta\right]\right\},(k=x, y)
\end{aligned}
$$

where $\bar{u}_{k f}\left(\mathbf{x}_{\perp}, 0\right)(k=x, y)$ represents the free field horizontal displacement, $\quad \bar{U}_{k^{\prime} k}^{(G)}\left(r_{i j}, 0, \zeta\right), \quad \bar{U}_{z k}^{(G)}\left(r_{i j}, 0, \zeta\right)$ $\left(k, k^{\prime}=x, y\right)$ denotes the horizontal displacement at the surface point $\mathbf{x}_{\perp}\left(\mathbf{x}_{\perp}=x \mathbf{i}+y \mathbf{j}\right)$ due to a unit patch horizontal and vertical load applied at $\Pi_{\xi}^{(j)}$, respectively.

For the close of the system, additional equations for $\bar{u}_{k^{*}}^{p(i)}(0)(k=x, y ; i=1,2, \ldots \ldots, m)$ are needed. Supposing that the horizontal displacement of the i-th fictitious pile top is equal to the horizontal displacement of the extended halfspace at the i-th pile top, supplementary equations for $\bar{u}_{k^{*}}^{p(i)}(0) \quad(k=x, y ; i=1,2, \ldots \ldots, m)$ in (46) can be obtained via (48) by setting $\mathbf{x}_{\perp}$ coincide with the center of the $\mathrm{i}$-th pile top. Thus, using (30)-(36) and (48), the following supplementary equations for $\bar{u}_{k^{*}}^{p(i)}(0)$ are derived

$$
\begin{aligned}
& \sum_{j=1}^{m(j \neq i)}\left[-\int_{0}^{L} \bar{N}_{*}^{(j)}(\zeta) \frac{\partial \bar{U}_{z k}^{(G)}\left(r_{i j}, 0, \zeta\right)}{\partial \zeta} d \zeta\right. \\
& \left.+\int_{0}^{L} \bar{N}_{*}^{(j)}(\zeta) \bar{\Lambda}_{i j}^{(c)}(0, \zeta) d \zeta+\bar{u}_{z^{*}}^{p(j)}(0) \bar{\Lambda}_{i j}^{(d)}(0)\right]
\end{aligned}
$$




$$
\begin{aligned}
& +\sum_{k^{\prime}=x, y}\left\{\sum _ { j = 1 } ^ { m } \left[\int_{0}^{L} \bar{Q}_{k^{\prime *}}^{(j)}(\zeta) \frac{\partial \bar{U}_{k^{\prime} k}^{(G)}\left(r_{i j}, 0, \zeta\right)}{\partial \zeta} d \zeta\right.\right. \\
& \left.\left.+\int_{0}^{L} \bar{\kappa}_{i j}^{(a)}(\zeta) \bar{U}_{k^{\prime} z}^{(G)}\left(r_{i j}, 0, \zeta\right) d \zeta+\bar{\theta}_{k^{\prime *}}^{p(j)}(0) \bar{\Gamma}_{i j}^{(c)}(z)\right]\right\} \\
& +\sum_{j=1}^{m} \bar{u}_{k^{*}}^{p(j)}(0)\left[\bar{\Gamma}_{i j}^{(d)}(z)-\delta_{i j}\right]+\sum_{j=1}^{m} \bar{u}_{k^{\prime *}}^{p(j)}(0) \bar{\Gamma}_{i j}^{(d)}(z) \\
& =-\bar{u}_{k f}^{(i)}(0), \\
& (i=1,2, \ldots, m, k=x, y)
\end{aligned}
$$

where

$$
\begin{aligned}
& \bar{\Lambda}_{i j}^{(c)}(z, \zeta)=\frac{\rho_{p^{*}}^{(j)} \omega^{2}}{E_{p^{*}}^{(j)}} \int_{\zeta}^{L} \bar{U}_{z k}^{(G)}\left(r_{i j}, z, \eta\right) d \eta,(k=x, y) \\
& \bar{\Lambda}_{i j}^{(d)}(z)=\rho_{p^{*}}^{(j)} A^{(j)} \omega^{2} \int_{0}^{L} \bar{U}_{z k}^{(G)}\left(r_{i j}, z, \eta\right) d \eta,(k=x, y) \\
& \bar{\Gamma}_{i j}^{(c)}(z)=\rho_{p^{*}}^{(j)} A^{(j)} \omega^{2} \int_{0}^{L} \zeta \bar{U}_{k^{\prime} k}^{(G)}\left(r_{i j}, z, \zeta\right) d \zeta, \\
& \left(k, k^{\prime}=x, y\right) \\
& \bar{\Gamma}_{i j}^{(b)}(z)=\rho_{p^{*}}^{(j)} A^{(j)} \omega^{2} \int_{0}^{L} \bar{U}_{k^{\prime} k}^{(G)}\left(r_{i j}, z, \zeta\right) d \zeta, \\
& \left(k, k^{\prime}=x, y\right)
\end{aligned}
$$

\section{DEFINITION OF THE AMPLITUDE REDUCTION RATIO}

To assess the isolation vibration effect of pile rows, the amplitude reduction ratio $A_{r}$, which is the ratio between the amplitude of the vertical displacement of the half-space in the presence of the pile rows and that of the free field solution, is defined as follows

$A_{r}=\frac{\left|\bar{u}_{z s}\left(\mathbf{x}_{\perp}, z=0\right)\right|}{\left|\bar{u}_{z}^{(f)}\left(\mathbf{x}_{\perp}, z=0\right)\right|}$

where $\mathbf{x}_{\perp}=x \mathbf{i}+y \mathbf{j},\left|\bar{u}_{z s}\left(\mathbf{x}_{\perp}, z=0\right)\right|,\left|\bar{u}_{z}^{(f)}\left(\mathbf{x}_{\perp}, z=0\right)\right|$ is the amplitude of the vertical displacement for the half-space with the pile rows and the amplitude of the vertical displacement for the free field solution. The displacement $\left|\bar{u}_{z}^{(f)}\left(\mathbf{x}_{\perp}, z=0\right)\right|$ can be obtained by performing the inverse Hankel transform on the frequency-wavenumber domain solution of the vertical displacement in Section 3.

Woods [26] proposed an average amplitude reduction ratio $\bar{A}_{r}$ for assessing the isolation vibration effect. The average amplitude reduction ratio $\bar{A}_{r}$ is defined as follows:

$$
\bar{A}_{r}=\frac{1}{A} \int_{A} A_{r} d A
$$

where $A$ is a rectangle with its width and length determined by the wavelength of the Rayleigh wave and the width of pile rows.

\section{NUMERICAL RESULTS AND DISCUSSIONS}

The integral equations accounting for the vertical and horizontal interaction between pile rows and the half-space can be solved numerically. The methodology for solving integral equations (39), (46) was detailed in [25]. To show the main idea for solving the second kind of Fredholm integral equations in this paper, the Fredholm integral equations in this paper is denoted by the following simple form

$$
a(z) \phi(z)+\int_{0}^{L} \phi(\xi) \frac{\partial \varepsilon(\xi, z)}{\partial \xi} d \xi=f(z), 0 \leq z \leq L
$$

where $a(z), f(z)$ are known functions and should be continuous within the interval $0 \leq z \leq L, \phi(\xi)$ is the unknown function to be determined, $\partial \varepsilon(\xi, z) / \partial \xi$ is the kernel function for the integral equation. As in this study, $\varepsilon(\xi, z)$ takes different values at $\xi=z^{-}$and $\xi=z^{+}$, integral equation (53) is one kind of weakly singular integral equation. Due to the discontinuity of $\partial \varepsilon(\xi, z) / \partial \xi$ at $z$, thus, it is appropriate to divide the integral in (53) into two parts at point $z$, which leads to

$\int_{0}^{L} \phi(\xi) \frac{\partial \varepsilon(\xi, z)}{\partial \xi} d \xi=\int_{0}^{z^{-}} \phi(\xi) \frac{\partial \varepsilon(\xi, z)}{\partial \xi} d \xi+\int_{z^{+}}^{L} \phi(\xi) \frac{\partial \varepsilon(\xi, z)}{\partial \xi} d \xi$

To implement the quadrature approach, the interval $0 \leq z \leq L$ is divided into $\mathrm{N}$ segments evenly. And within each segment $z_{j} \leq \xi \leq z_{j+1}$ of the $\mathrm{N}$ segments, $\phi(\xi)$ is approximated by

$\phi(\xi)=\left(\phi\left(z_{j}\right)+\phi\left(z_{j+1}\right)\right) / 2, z_{j} \leq \xi \leq z_{j+1}$

Substitution of (55) into the integration over the interval $z_{j} \leq \xi \leq z_{j+1}$, one has

$$
\begin{aligned}
& \int_{z_{j}}^{z_{j+1}} \phi(\xi) \frac{\partial \varepsilon(\xi, z)}{\partial \xi} d \xi=(1 / 2)\left[\phi\left(z_{j}\right)\right. \\
& \left.+\phi\left(z_{j+1}\right)\right] \int_{z_{j}}^{z_{j+1}} \frac{\partial \varepsilon(\xi, z)}{\partial \xi} d \xi \\
& =(1 / 2)\left[\phi\left(z_{j}\right)+\phi\left(z_{j+1}\right)\right]\left[\varepsilon\left(z_{j+1}, z\right)-\varepsilon\left(z_{j}, z\right)\right], \\
& z_{j} \leq \xi \leq z_{j+1}
\end{aligned}
$$

Using (56) to evaluate the integrations over the $N$ subintervals in (53), then, the integral equation (53) is reduced to the following discrete form

$$
\begin{aligned}
& a\left(z_{i}\right) \phi\left(z_{i}\right)+\sum_{j=1}^{N}(1 / 2)\left[\phi\left(z_{j}\right)\right. \\
& \left.+\phi\left(z_{j+1}\right)\right]\left[\varepsilon\left(z_{j+1}, z_{i}\right)-\varepsilon\left(z_{j}, z_{i}\right)\right]=f\left(z_{i}\right), \\
& i=1,2,3 \ldots N+1
\end{aligned}
$$


It is worth stressing that when $j+1$ in the above equation equal to $i$, then, $\varepsilon\left(z_{j+1}, z_{i}\right)$ will assume the values $\varepsilon\left(z_{i}^{-}, z_{i}\right)$, as the interval $z_{j} \leq \xi \leq z_{j+1}$ infinitely approaches the point $z_{i}$ form the above; on the other hand, when $j=i$, then, $\varepsilon\left(z_{j}, z_{i}\right)$ should take the value $\varepsilon\left(z_{i}^{+}, z_{i}\right)$ as the interval $z_{j} \leq \xi \leq z_{j+1}$ is infinitely close to the point $z_{i}$ from the below.

Discrete formula (57) can be used to reduce the integral equations (39), (46) to linear algebraic equations. After the numerical solution of the linear algebraic equations, the vertical displacement of the half-space can be obtained via (41) and the amplitude reduction ratio can be evaluated by (51) and (52).

To verify the proposed method, a special case of our model will be compared with an existing result in Sec. 6.1. Also, some numerical examples and corresponding analysis are given in Sec. 6.2 and Sec. 6.3.

\subsection{Comparison of our Result with an Existing Result}

As the first example, a special case of our solution is compared with an existing result. In our solution, a single pile row with eight piles embedded in the poroelastic halfspace is used as the passive vibration isolation system again a harmonic vertical patch load. The parameters for each pile assume the following values: $d=1.0 \mathrm{~m}, L=5.0 \mathrm{~m}$, $E_{p}=4.526 \times 10^{10} \mathrm{~N} / \mathrm{m}^{2}, \quad \rho_{p}=2.35 \times 10^{3} \mathrm{~kg} / \mathrm{m}^{3}$. The net spacing between two neighboring piles is $s=0.5 \mathrm{~m}$. The vibration source is a harmonic vertical uniform patch load with magnitude $Q_{F}=200 \mathrm{kN} / \mathrm{m}^{2}$ and applied over a circular area with the diameter $D=0.8 \mathrm{~m}$. Its frequency is $f=50 \mathrm{~Hz}$. The distance between the pile row and the center of the vibration source is $d_{s}=7.5 \mathrm{~m}$ (see Fig. 1). To reduce the poroelastic half-space to a quasi-elastic halfspace, the parameters $M, m, \alpha, b_{p}, \rho_{f}$ are set to be 0.0001 as in [27]. It should be noted that for an elastic medium there are singularities in the path of the integration when evaluating the fundamental solution, which makes the numerical evaluation of the integral a formidable task. However, some researchers tend to evaluate this kind of integrals by use of a special viscoelastic model. In the viscoelastic model, the material damping is taken into account by using complex Lame constants i.e. $\mu=\mu_{0}\left(1+\mathrm{i} \beta_{s}\right)$ and $\lambda=\lambda_{0}\left(1+\mathrm{i} \beta_{s}\right)$, where $\beta_{s}$ denotes the damping ratio. In this paper, we use a damping ratio of $\beta_{s}=0.05$ for the half-space and $\mu_{0}=1.32 \times 10^{8} \mathrm{~N} / \mathrm{m}^{2}, \lambda_{0}=1.32 \times 10^{8} \mathrm{~N} / \mathrm{m}^{2}, \rho_{s}=1.75 \times 10^{3} \mathrm{~kg} / \mathrm{m}^{3}$. According to [6], the reference Rayleigh wavelength for the quasi elastic half-space is $\lambda_{R}=5.0 \mathrm{~m}$.

Fig. (4) shows the contour of the amplitude reduction ratio $A_{r}$ for the single pile row embedded in the quasi elastic half-space according to the present model. It can be observed that the amplitude reduction ratio $A_{r}$ right behind the pile rows is much smaller than those at other areas. For further comparison, the average amplitude reduction ratio $\bar{A}_{r}$ for the quasi elastic half-space is calculated, which is 0.718 , while the result of Kattis [6] for the elastic half-space is 0.712 . The difference between the present solution and that of Kattis' [6] is only $0.842 \%$.

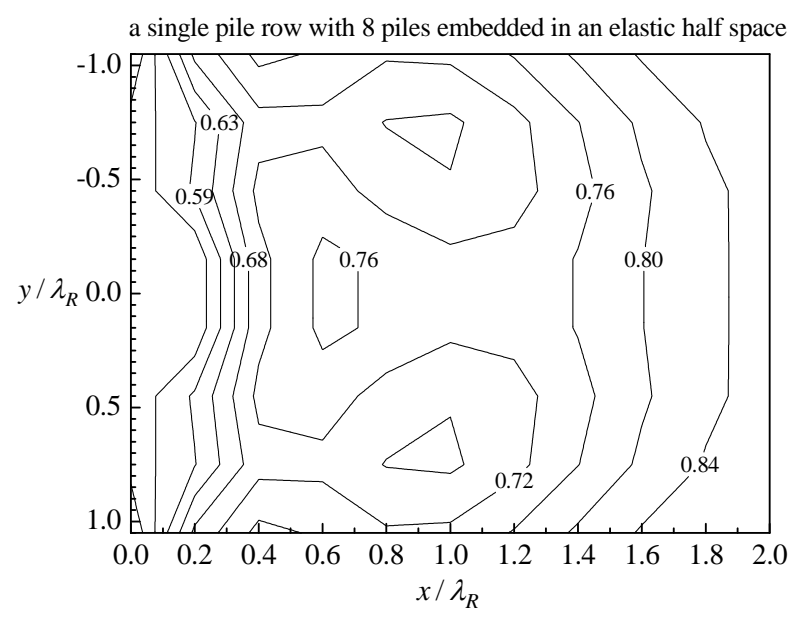

Fig. (4). The contour of the amplitude reduction ratio $A_{r}$ for a single pile row with eight piles used as the passive system.

\subsection{Numerical Simulation of the Vibration Isolation using a Single Pile Row}

In this section, the passive isolation vibration system is a single pile row with nine piles embedded in a poroelastic half-space. The vibration source is a harmonic vertical uniform patch load with the magnitude $Q_{F}$ and applied over a circular area with the diameter $D$ and its frequency is $f$. The reference Rayleigh wavelength for the half-space is $\lambda_{R}=5.0 \mathrm{~m}$. The parameters for the poroelastic half-space, pile rows and the vibration source are given in Table $\mathbf{1}$.

In the following, the influences of Biot's parameter $(M)$, Young's modulus of the pile ( $\left.E_{p}\right)$, the pile length $(L)$ and the net spacing between two neighboring piles in a pile row $(s)$ on the vibration isolation effect will be discussed. Note that when the influence of one parameter is examined, all the other parameters will remain fixed and take the typical values as given in Table $\mathbf{1}$.

\subsubsection{The Effect of Biot's Parameter (M)}

To study the influence of Biot's parameter $(M)$ on the vibration isolation effect of the pile row, Biot's parameter $(M)$ takes the following three different values: $M=2.0 \times 10^{8} \mathrm{~Pa}, 2.0 \times 10^{10} \mathrm{~Pa}$ and $2.0 \times 10^{12} \mathrm{~Pa}$, respectively. The other parameters for the piles, the vibration source and the poroelastic half-space assume the values as given in Table $\mathbf{1}$.

Fig. 5(a)-(c) shows the amplitude reduction ratio $A_{r}$ on the surface for the poroelastic half-space for 
Table 1. The Parameters for the Poroelastic Half-Space, Pile Rows and the Vibration Source in Sec. 6.2

\begin{tabular}{|c|c|}
\hline Parameters & Values \\
\hline$\lambda(\mathrm{Pa})$ & $2.6 \times 10^{7}$ \\
\hline$\rho_{s}\left(\mathrm{~kg} / \mathrm{m}^{3}\right)$ & $2.6 \times 10^{3}$ \\
\hline$\rho_{f}\left(\mathrm{~kg} / \mathrm{m}^{3}\right)$ & $1.0 \times 10^{3}$ \\
\hline$a_{\infty}$ & 2.0 \\
\hline$\alpha$ & 0.97 \\
\hline$M\left(\mathrm{~N} / \mathrm{m}^{2}\right)$ & $2.8 \times 10^{8}$ \\
\hline$b_{p}(\mathrm{~kg} /(\mathrm{m} 3 . \mathrm{s}))$ & $1.0 \times 10^{10}$ \\
\hline$Q_{F}$ (the magnitude of the harmonic vertical uniform load) $(\mathrm{kN})$ & 100 \\
\hline $\begin{array}{l}d_{s} \text { (the distance between the center of the first pile row and the vibration } \\
\text { source })(\mathrm{m})\end{array}$ & 7.5 \\
\hline$d$ (the diameter of the piles) (m) & 0.8 \\
\hline$L$ (the pile length) (m) & 10.0 \\
\hline$E_{p} / E_{s}$ & 50 \\
\hline$\rho_{p}$ (the density of the piles) $\left(\mathrm{kg} / \mathrm{m}^{3}\right)$ & $3.9 \times 10^{3}$ \\
\hline$s(\mathrm{~m})$ & 0.8 \\
\hline$q$ (the spacing between the adjacent pile rows) (m) & 0.4 \\
\hline
\end{tabular}

$M=2.0 \times 10^{8} \mathrm{~Pa}, 2.0 \times 10^{10} \mathrm{~Pa}$ and $2.0 \times 10^{12} \mathrm{~Pa}$, respectively. Fig. (6) illustrates the amplitude reduction ratio $A_{r}$ within the range $x / \lambda_{R}=0 \sim 2$ and $y=0$ when Biot's parameter takes the above three values. The average amplitude reduction ratio $\bar{A}_{r}$ for the three cases of Biot's parameter $(M)$ are as follows: for $M=2.0 \times 10^{8} \mathrm{~Pa}, \bar{A}_{r}=0.6586$; for $M=2.0 \times 10^{10} \mathrm{~Pa}, \bar{A}_{r}=0.6176$; for $M=2.0 \times 10^{12} \mathrm{~Pa}$, $\bar{A}_{r}=0.6166$. One can see that the average amplitude reduction ratio $\bar{A}_{r}$ decreases slightly with the increasing $M$, which shows that Biot's parameter $M$ has a small effect on the vibration isolation effect of the pile row.

\subsubsection{The Effect of Young's Modulus of the Piles $\left(E_{p}\right)$}

Young's modulus $\left(E_{p}\right)$ of the pile is an important parameter for the design of the pile row isolation system. Herein, the effect of Young's modulus of the piles on the vibration isolation effect will be investigated. In this example, Young's modulus of the piles assumes the following four values: $E_{p} / E_{s}=50,100,200$ and 500, respectively, with $E_{s}$ fixed and given in Table 1.

Fig. 7(a)-(c) shows the variation of the amplitude reduction ratio $A_{r}$ on the surface of the poroelastic half-space for
$E_{p} / E_{s}=100,200$ and 500, respectively. Note that the result for the case of $E_{p} / E_{s}=50$ has already been presented in Sec.6.2.1 (see Fig. 5(a)). Fig. (8) illustrates the amplitude reduction ratio $A_{r}$ within the range $x / \lambda_{R}=0 \sim 2$ and $y=0$ for the poroelastic half-space when $E_{p} / E_{s}$ takes 50, 100, 200 and 500, respectively. The average amplitude reduction ratio $\bar{A}_{r}$ for the four cases of modulus ratios are: for $E_{p} / E_{s}=50, \quad \bar{A}_{r}=0.6586 ; \quad$ for $\quad E_{p} / E_{s}=100$, $\bar{A}_{r}=0.5926 ;$ for $E_{p} / E_{s}=200, \quad \bar{A}_{r}=0.5644$; for $E_{p} / E_{s}=500, \bar{A}_{r}=0.5489$. One can be see clearly that the average amplitude reduction ratio $\bar{A}_{r}$ decreases with the increasing $E_{p} / E_{s}$. Moreover, when $E_{p} / E_{s} \geq 200, \bar{A}_{r}$ only decreases slightly with the increasing $E_{p} / E_{s}$. As a result, it can be concluded that a stiffer pile row will produce a better isolation vibration effect.

\subsubsection{The Effect of the Pile Length}

The influence of the pile length on the isolation vibration effect is examined in this section. The pile length takes three different values: $L=5.0 \mathrm{~m}, 10.0 \mathrm{~m}$ and $15.0 \mathrm{~m}$, respectively. 


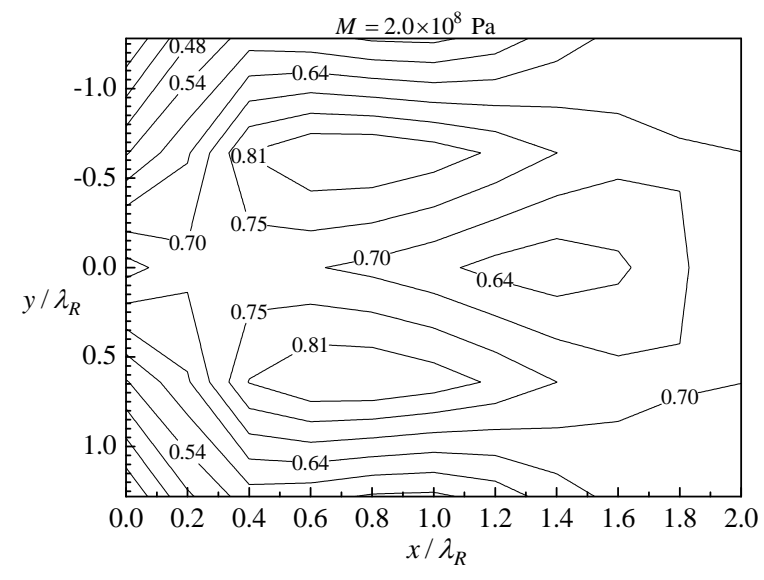

a

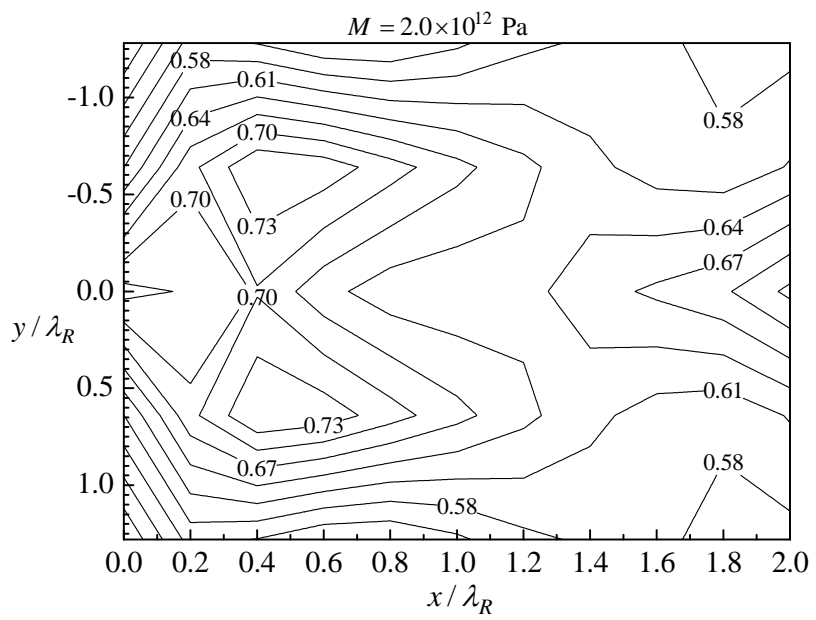

c

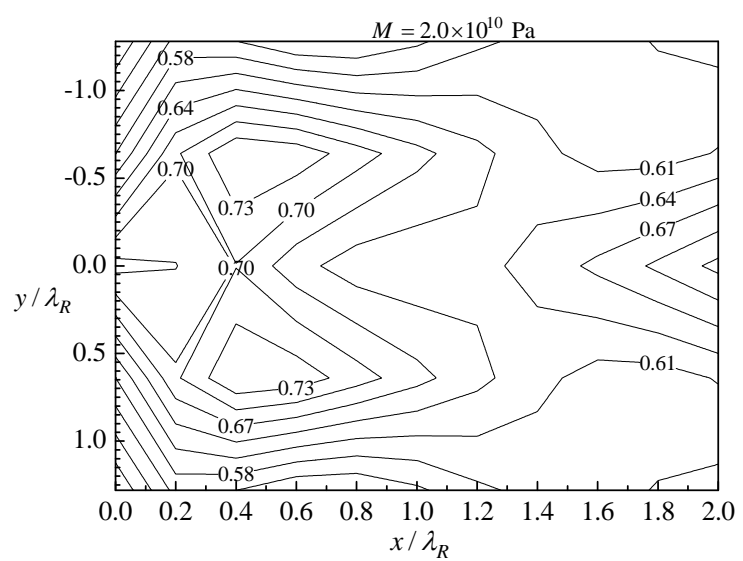

b

Fig. (5). The contour of the amplitude reduction ratio $A_{r}$ for a single pile row with nine piles embedded in the poroelastic half space for different values of Biot's parameter $M$ : (a) $M=2.0 \times 10^{8} \mathrm{~Pa} ;$ (b) $M=2.0 \times 10^{10} \mathrm{~Pa} ;$ (c) $M=2.0 \times 10^{12} \mathrm{~Pa}$.

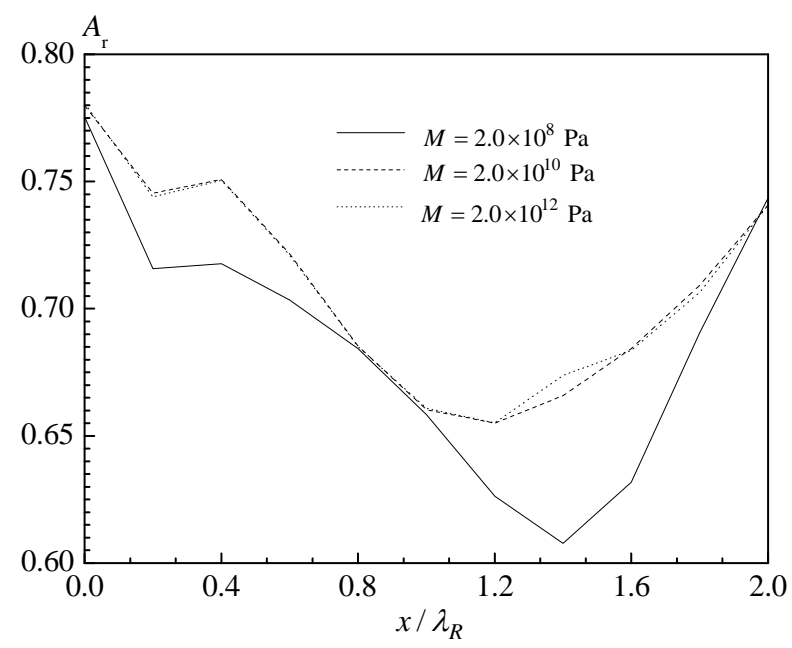

Fig. (6). Variation of the amplitude reduction ratio $A_{r}$ for a single pile row with nine piles embedded in the poroelastic half space within the range $0 \leq x / \lambda_{R} \leq 2$ and $y=0$ when Biot's parameter $(M)$ takes the three different values. 


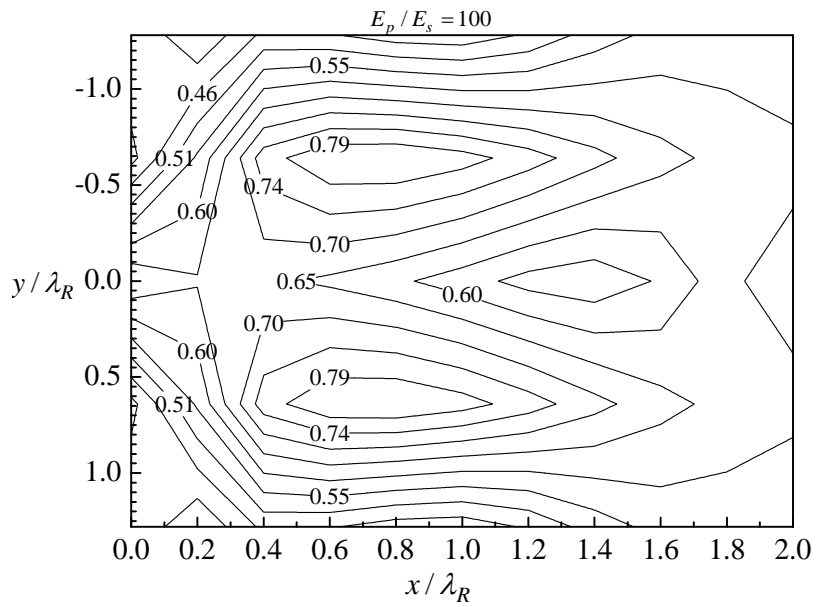

$\mathbf{a}$

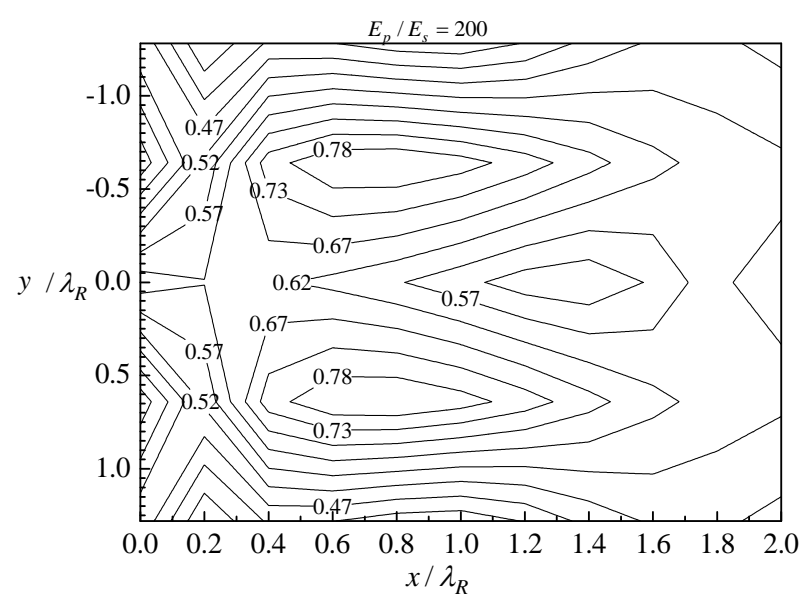

b

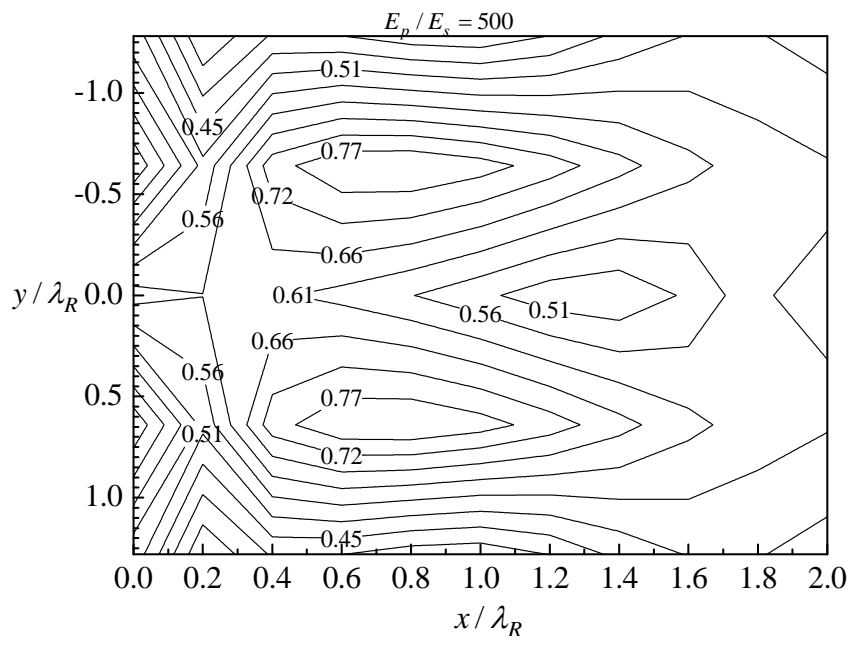

c

Fig. (7). The contour of the amplitude reduction ratio $A_{r}$ for a single pile row with nine piles embedded in the poroelastic half space for different values of the ratio of Young's modulus between the piles and the poroelastic half space: (a) $E_{p} / E_{s} 100 ;$ (b) $E_{p} / E_{s}=200 ;$ (c) $E_{p} / E_{s} 500$.

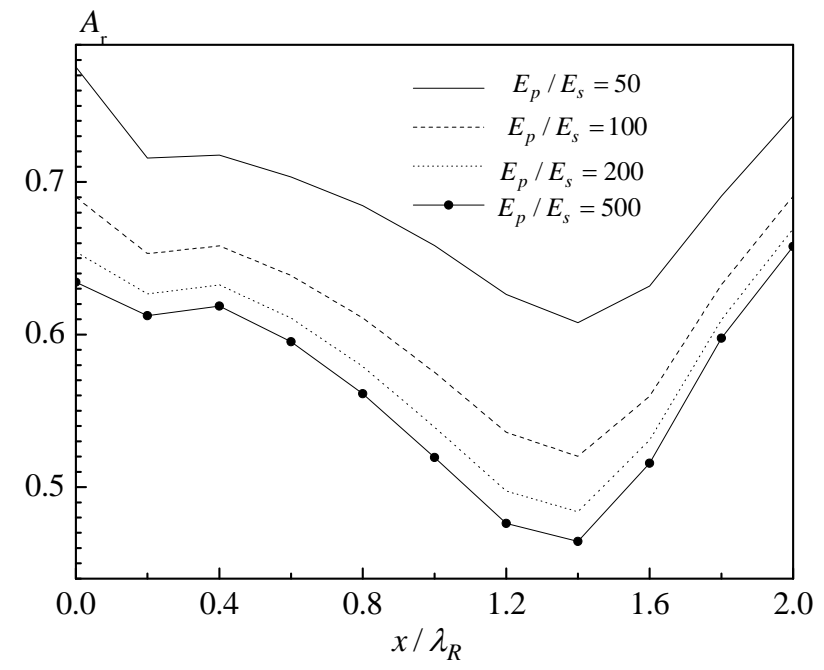

Fig. (8). The amplitude reduction ratio $A_{r}$ within the range $0 \leq x / \lambda_{R} \leq 2$ and $y=0$ when the ratio of Young's modulus between the piles and the poroelastic half takes the values 50,100,200 and 500, respectively. 
Figs. 9(a), (b) show the variation of the amplitude reduction ratio $A_{r}$ on the surface of the poroelastic half-space for $L=5.0 \mathrm{~m}$ and $15.0 \mathrm{~m}$, respectively. The result for the case of $L=10.0 \mathrm{~m}$ is illustrated in Fig. 5(a). Fig. (10) illustrates that the amplitude reduction ratio $A_{r}$ within $x / \lambda_{R}=0 \sim 2$ and $y=0$ for the poroelastic half-space when the pile length takes $L=5.0 \mathrm{~m}, 10.0 \mathrm{~m}$ and $15.0 \mathrm{~m}$, respectively. The average amplitude reduction ratios $\bar{A}_{r}$ for $L=5.0 \mathrm{~m}, 10.0 \mathrm{~m}$ and $15.0 \mathrm{~m}$ equal to $0.8653,0.6586,0.6884$, respectively. It can be seen that the length of piles has a significant influence on the average amplitude reduction ratios $\bar{A}_{r}$ when $L \leq 10.0 \mathrm{~m}$. However, when $L$ is larger than $10.0 \mathrm{~m}$, with increase of the pile length, $\bar{A}_{r}$ only change slightly, which agrees with the result in [7].

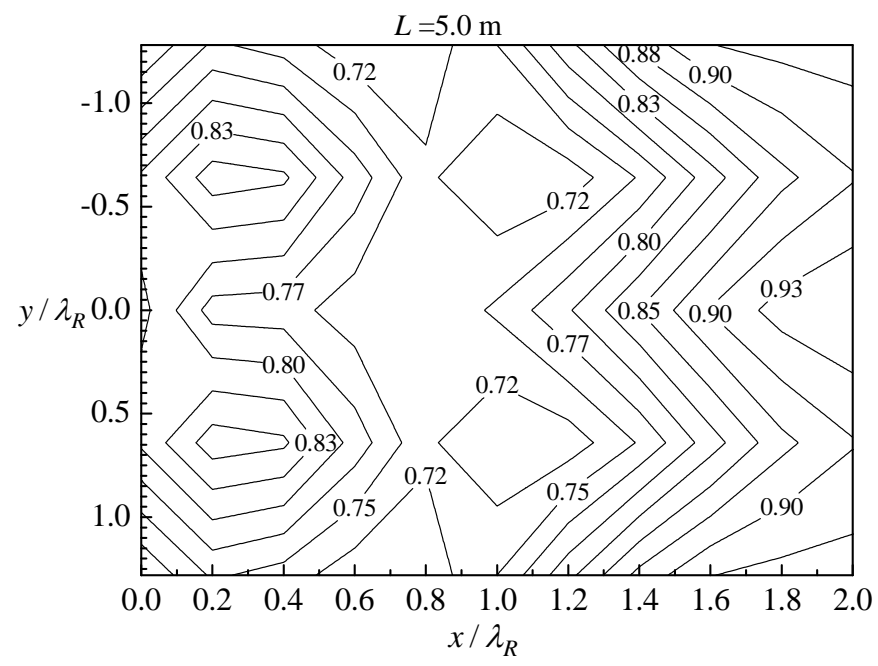

a

\subsubsection{The Effect of the Pile-Pile Net Spacing}

The net spacing between two neighboring piles in a pile row is an important parameter for the design of pile rows for vibration isolation. In this section, the value of $s$ takes the following three values: $0.8 \mathrm{~m}, 1.2 \mathrm{~m}$ and $1.6 \mathrm{~m}$, respectively. The other parameters for the pile, the poroelastic halfspace and the vibration source take the values as given in Table 1.

Fig. 11(a), (b) shows the variation of the amplitude reduction ratio $A_{r}$ on the surface of the poroelastic half-space for $S=1.2 \mathrm{~m}$ and $1.6 \mathrm{~m}$, respectively. The result for the case of $S=0.8 \mathrm{~m}$ is given in Fig. 5(a).

Fig. 5(a) and Fig. (11) clearly indicate the decay of the isolation vibration effect behind the pile row with the in-

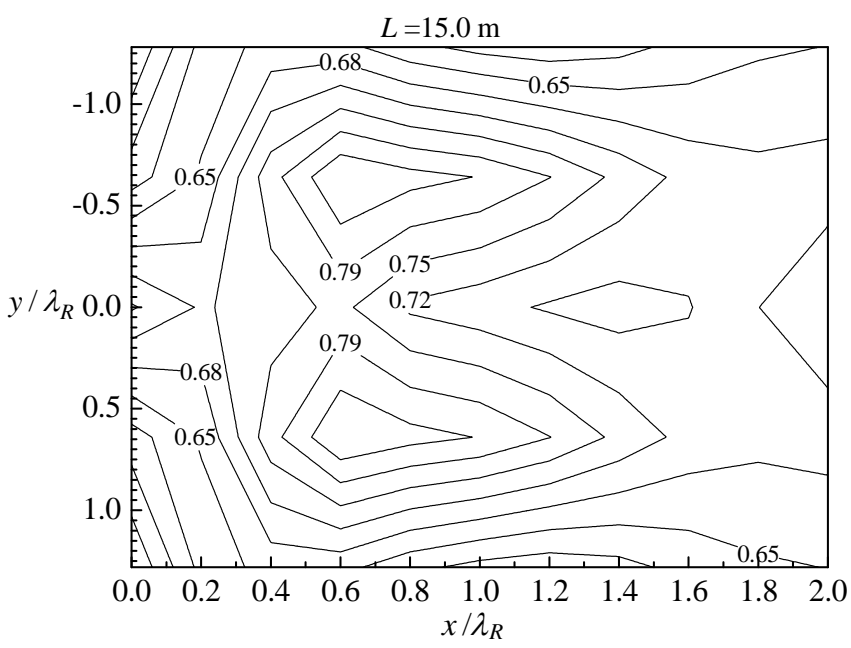

b

Fig. (9). The contour of the amplitude reduction ratio $A_{r}$ for the single pile row with nine piles for different values of the pile length: (a) $L=5.0 \mathrm{~m}$; (b) $L=15.0 \mathrm{~m}$.

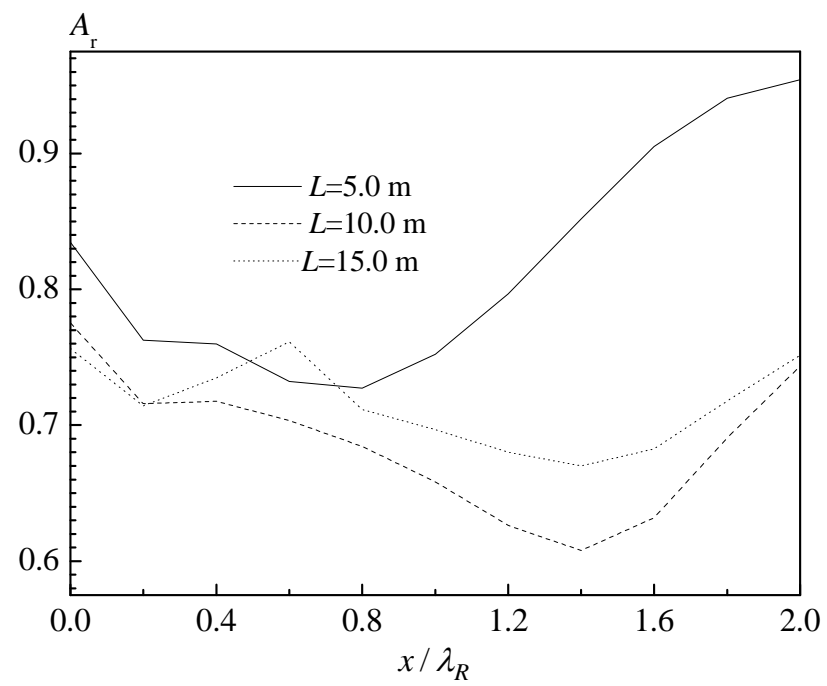

Fig. (10). The amplitude reduction ratio $A_{r}$ within the range $0 \leq x / \lambda_{R} \leq 2$ and $y=0$ when the pile length takes the values $L=5.0 \mathrm{~m}$, $10.0 \mathrm{~m}$ and $15.0 \mathrm{~m}$, respectively. 
creasing pile net spacing $s$. The average amplitude reduction ratios for the three cases of net spacing are: for $s=0.8 \mathrm{~m}$, $\bar{A}_{r}=0.6586$; for $s=1.2 \mathrm{~m}, 0.7133$; for $s=1.6 \mathrm{~m}, 0.7486$, which shows that the average amplitude reduction ratio $\bar{A}_{r}$ has a considerable increase with the increasing net spacing $s$. Thus, it can be concluded that a better isolation vibration effect can be realized by smaller net pile spacing.

\subsection{Numerical Simulation of the Vibration Isolation using Multiple Pile Rows}

In this section, the vibration isolation effect of multiple pile rows is investigated. The vibration source is a harmonic vertical uniform patch load with the magnitude $Q_{F}$ and the frequency $f$. The patch load is applied over a circular domain with the diameter $D$. A single pile row with seven piles, two pile rows with seven and six piles and three pile rows with seven, six and seven piles, respectively are considered in this example (Fig. 12), respectively. The net spacing between two neighboring piles in a pile row is $s=0.2 \mathrm{~m}$. The other parameters for the pile, the poroelastic half-space and the vibration source are given in Table $\mathbf{1}$ except that $d=0.4 \mathrm{~m}$ and $E_{p}=1.735 \times 10^{9} \mathrm{~N} / \mathrm{m}^{2}$. The reference Rayleigh wavelength is assumed to be $\lambda_{R}=5.0 \mathrm{~m}$.

In the following, the influences of the number of the pile rows, the net spacing between two neighboring piles and the spacing between neighboring pile rows on the vibration isolation effect are discussed. It is noted that when the influence of one parameter is examined, all the other parameters will remain unchanged and take the typical values as given above and in Table 1.

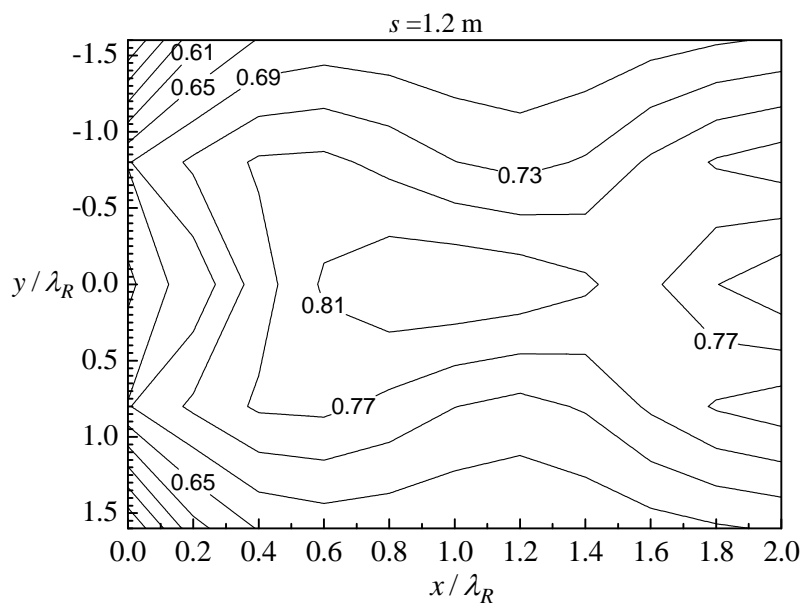

a

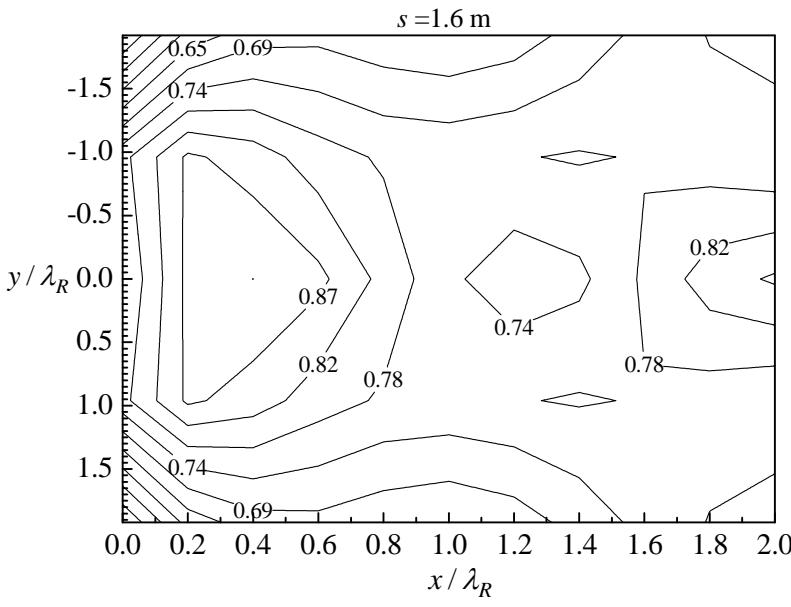

b

Fig. (11). The contour of the amplitude reduction ratio $A_{r}$ for the single pile row with nine piles for different values of the net spacing $(s)$ between two neighboring piles: (a) $s=1.2 \mathrm{~m}$; (b) $s=1.6 \mathrm{~m}$.

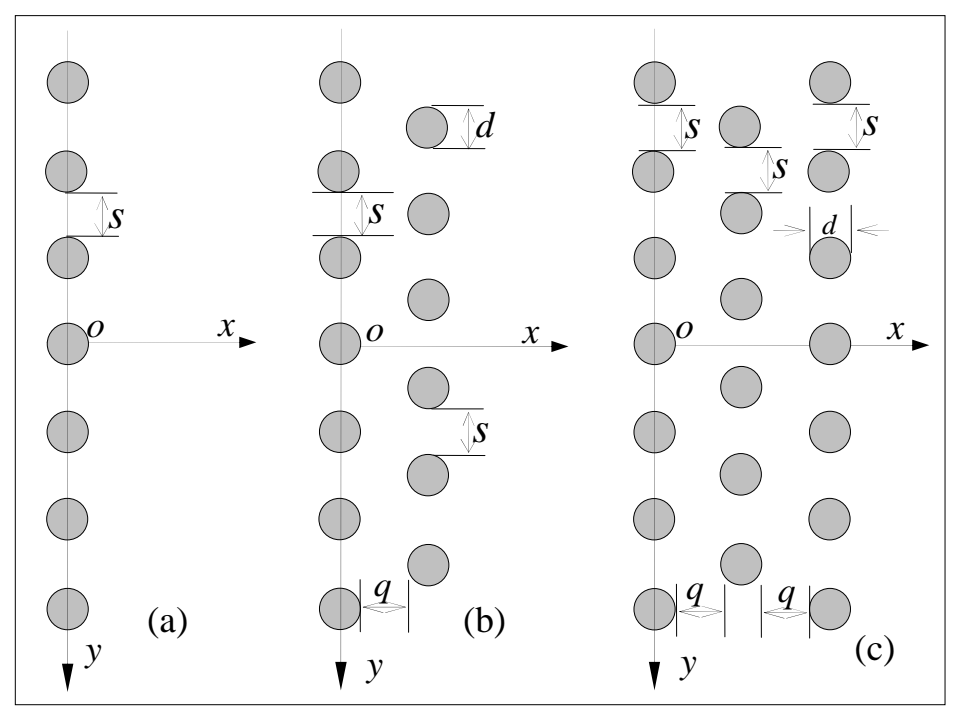

Fig. (12). Three kinds of pile rows used as vibration isolation system for the poroelastic half space subjected to a harmonic vertical load: (a) a single pile row with seven piles; (b) two pile rows with seven and six piles, respectively; (c) three piles rows with seven, six and seven piles, respectively. 


\subsubsection{The Effect of the Number of the Pile Rows}

In this example, we consider the following three cases of pile rows embedded in the poroelastic half-space: a single pile row with seven piles; two pile rows with seven and 6 piles, respectively; three pile rows with seven, six and seven piles, respectively (Fig. 12). All the parameters for the pile row, the poroelastic half-space and the circular patch load assume the values as given above and in Table $\mathbf{1}$.

Fig. 13(a)-(c) plot the variation of the amplitude reduction ratio $A_{r}$ on the surface of the poroelastic half-space for the three different kinds of the pile rows. The variation of the amplitude reduction ratios $A_{r}$ within $x / \lambda_{R}=0 \sim 2$ and $y=0$ for three cases of pile rows are also shown in Fig. (14).

As expected, it follows from Fig. (13) that the increase of the number of pile rows will enhance the isolation vibration effect of the pile rows. The average amplitude reduction ratios for the single pile row, the two piles rows, the three pile rows are $\bar{A}_{r}=0.7118,0.5408,0.4466$, respectively.

\subsubsection{The Effect of the Net Spacing Between Two Neigh- boring Piles}

In this section, we consider two pile rows embedded in the poroelastic half-space. The first and second pile row has seven and six piles, respectively (Fig. 12(b)). The net spacing between two neighboring piles in a pile row takes the following three different values: $s=0.2 \mathrm{~m}, 0.4 \mathrm{~m}, 0.8 \mathrm{~m}$, respectively, while the parameters for the poroelastic halfspace, the piles and the vibration source assume the typical values as given above and in Table $\mathbf{1}$.

Fig. 15 (a), (b) plot the amplitude reduction ratio $A_{r}$ on the surface of the poroelastic half-space for $s=0.4 \mathrm{~m}$ and $0.8 \mathrm{~m}$, respectively. Note that the result of $A_{r}$ for the two pile rows with $s=0.2 \mathrm{~m}$ is given in Fig. 13(b). For the two pile rows with $s=0.2 \mathrm{~m}, 0.4 \mathrm{~m}, 0.8 \mathrm{~m}$, respectively, the

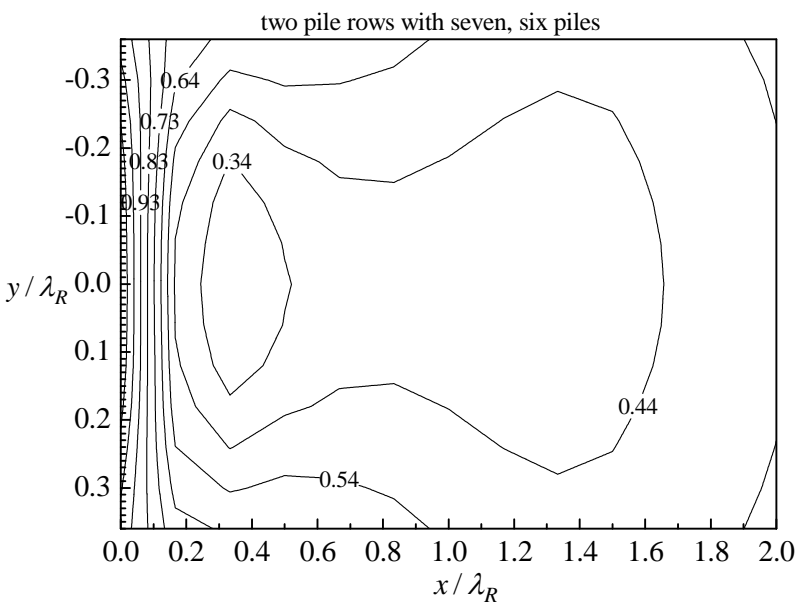

b

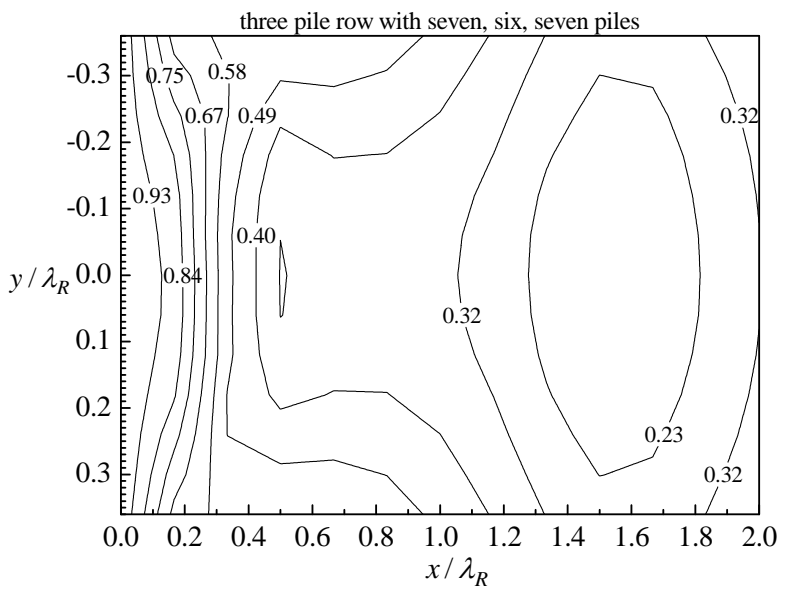

Fig. (13). The variation of the amplitude reduction ratio $A_{r}$ on the surface for the poroelastic half space for three different piles rows: (a) the single pile row with seven piles; (b) two pile rows with seven and six piles, respectively; (c) three piles rows with seven, six and seven piles, respectively. 
average amplitude reduction ratios are $\bar{A}_{r}=0.5408,0.6299$, 0.7583 , respectively. One can see that the average amplitude reduction ratio $\bar{A}_{r}$ for multiple pile rows increase with the increasing net spacing $s$. Thus, it can be concluded that the pile rows with a smaller separation between neighboring piles is generally more efficient in vibration isolation.

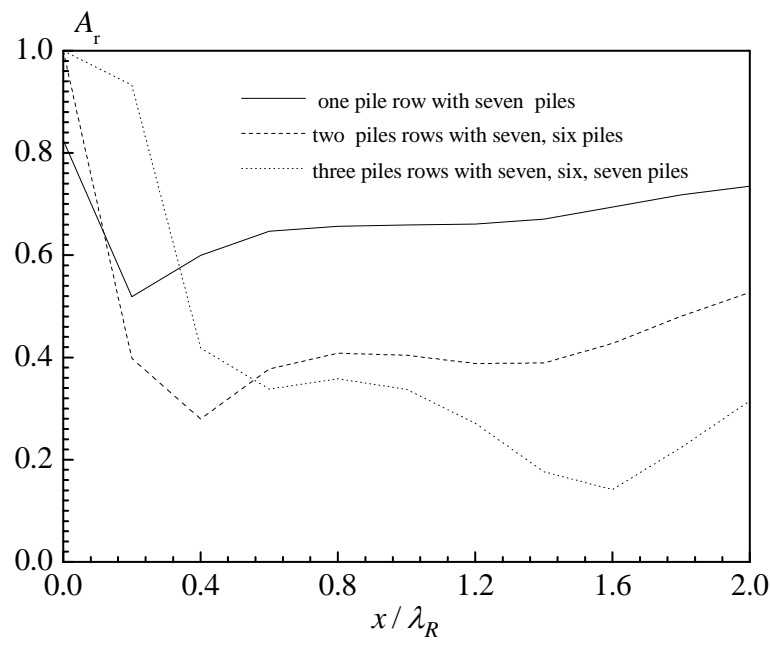

Fig. (14). The variation of the amplitude reduction ratio $A_{r}$ within the range $0 \leq x / \lambda_{R} \leq 2$ and $y=0$ for three kinds of pile rows.

\subsubsection{The Effect of the Spacing Between Two Adjacent Pile Rows}

To examine the effect of the spacing between neighboring pile rows, only two pile rows with the number of piles seven, six in the first and the second row, respectively are considered in this section (see Fig. 12(b)). The value of $q$ takes $0.4 \mathrm{~m}, 0.8 \mathrm{~m}$ and $1.2 \mathrm{~m}$, respectively, while the parameters for the poroelastic half-space, the piles and the vibration source are given as above and in Table $\mathbf{1}$.

Fig. 16(a), (b) depicts the amplitude reduction ratio $A_{r}$ on the surface of the half-space for $q=0.8 \mathrm{~m}$ and $1.2 \mathrm{~m}$ respectively. The result of $A_{r}$ for the case of $q=0.4 \mathrm{~m}$ is given in Fig. 13(b). For the two pile rows with $q=0.4 \mathrm{~m}$, $0.8 \mathrm{~m}$ and $1.2 \mathrm{~m}$, the average amplitude reduction ratios are $\bar{A}_{r}=0.5408,0.5482,0.5568$, respectively. From the result for the average amplitude reduction ratios and Fig. (16), one can observe that compared with other parameters, the space between two adjacent pile rows has a relatively smaller effect on the vibration isolation of the pile rows.

\section{CONCLUSIONS}

The numerical simulation of the isolation of the vibration due to a harmonic vertical load applied on the surface of a poroelastic half-space by pile rows has been carried out in this study. The second kind of Fredholm integral equations describing the dynamic interaction between the piles and the half space are obtained using Muki's method. The vibration isolation effect of pile rows is analyzed via the proposed semi-analytical model. As the semi-analytical model proposed in this paper avoids the discretization of the whole calculation domain, thus, it can save CPU time substantially for the current full 3-D problem. To investigate the vibration isolation effect of pile rows, the influence of Biot's parameter $(M)$, Young's modulus of the pile $\left(E_{p}\right)$, the pile length $(L)$, the spacing between two neighboring piles $(s)$ in a pile row are investigated via the semi-analytical model. Also, the influence of the number of the pile rows $(n)$ and the spacing $(q)$ between two adjacent pile rows are examined.

From the numerical results of this study, the following conclusions can be drawn:

1. The larger $M$ corresponds to a slightly better vibration isolation effect than the smaller $M$.

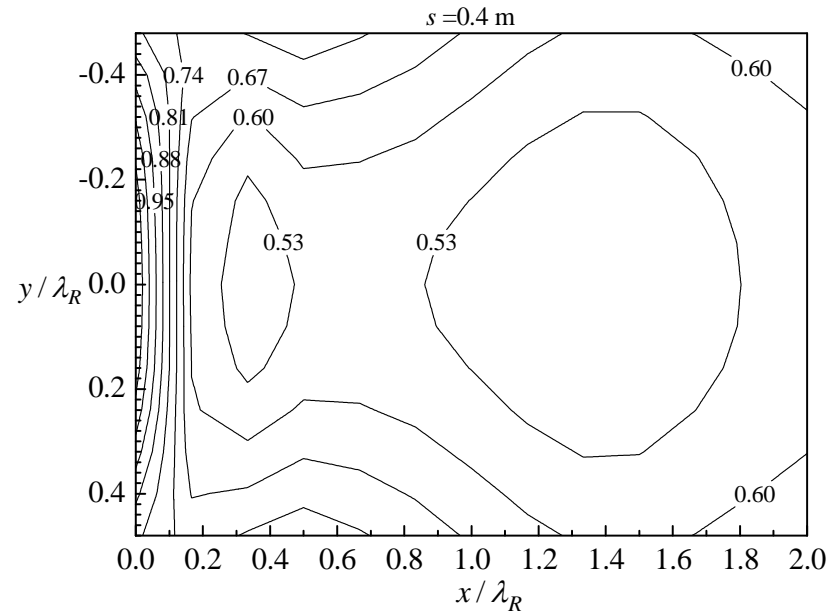

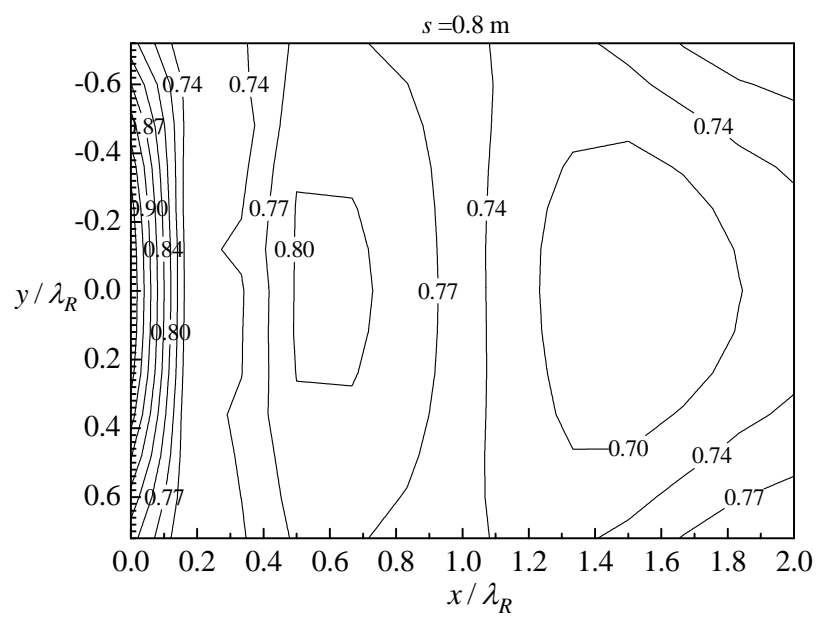

b

Fig. (15). The variation of the amplitude reduction ratio $A_{r}$ on the surface for the poroelastic half space for two pile rows with seven and six piles, respectively, for different values of the net spacing $(s)$ between two neighboring piles: (a) $s=0.4 \mathrm{~m} ;(\mathbf{b}) s=0.8 \mathrm{~m}$. 


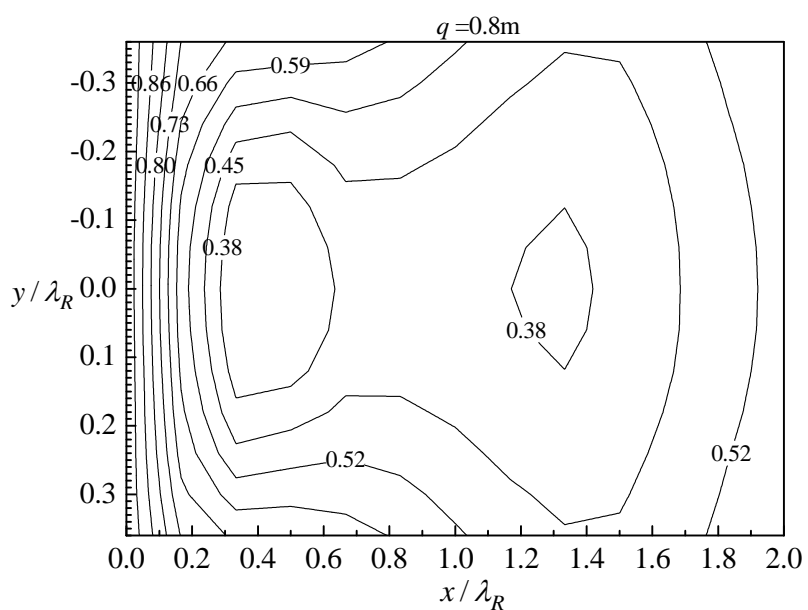

a

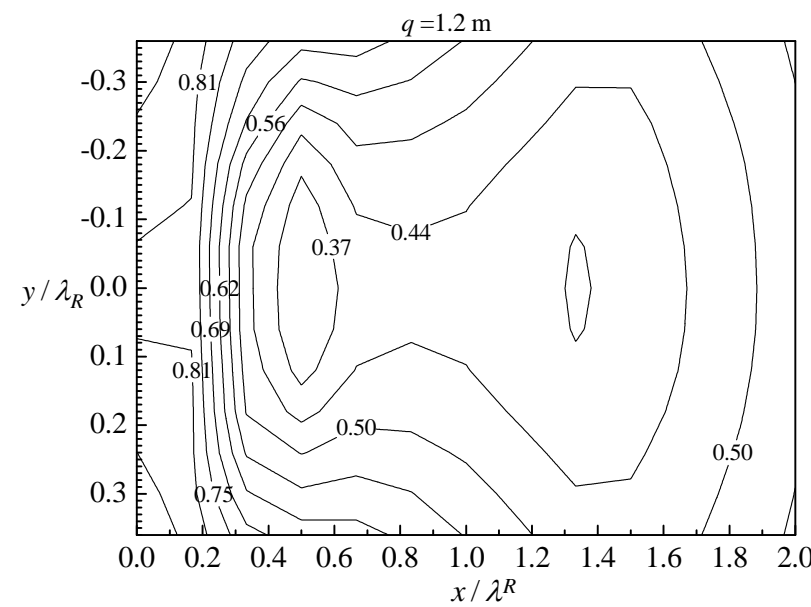

b

Fig. (16). The contour of the amplitude reduction ratio $A_{r}$ on the surface for the poroelastic half space for two pile rows with seven and six piles, respectively, for different spacing between two neighboring pile rows: (a) $q=0.8 \mathrm{~m}$; (b) $q=1.2 \mathrm{~m}$.

2. The length of the piles is a most important factor for the vibration isolation effect. Usually, longer piles will produce a better vibration isolation effect. The recommended optimal length of the piles for vibration isolation purpose ranges between $1.5 \sim 2.0 \lambda_{R}$, in which $\lambda_{R}$ is the wavelength of the Rayleigh wave of the porous halfspace.

3. Young's modulus $\left(E_{p}\right)$ of the piles also has significant influence on the effect of vibration isolation. Generally, the vibration isolation effect can be improved by increasing Young's modulus of the piles $\left(E_{p}\right)$.

4. The net spacing between two neighboring piles is also a key factor for the vibration isolation effect. Usually, smaller net pile spacing will result in a better vibration isolation effect.

5. Multiple pile rows tend to produce a better vibration isolation effect than a single pile row. Moreover, the spacing between the pile rows has a relatively smaller impact on the vibration isolation effect.

\section{ACKNOWLEDGMENTS}

The project is supported by the National Natural Science Foundation of China with grant number No. 50578071, 50969007. Also, the research is supported by the returned overseas scholar funding from Chinese Education Ministry. Besides, this research is also supported financially by State Key Laboratory for GeoMechanics and Deep Underground Engineering (No. SKLGDUE08012X).

\section{REFERENCES}

[1] K. Emad, and G. D. Manolis, "Shallow trenches and propagation of surface waves", J. Eng. Mech., ASCE, Vol. 111, pp. 279-282, 1985.

[2] D. E Beskos, G. Dasgupta, and I. G. Vardoulakis, "Vibration isolation using open or filled trench. Part 1: 2-D homogeneous", Comput. Mech., Vol. 1, pp. 43-63, 1986.
[3] B. Dasgupta, D. E. Beskos, and I. G. Vordouclakis, "Vibration isolation using open or filled trenches. Part 2: 3-D homogeneous soil", Comput. Mech., Vol. 6, pp. 129-142, 1990.

[4] J. Avilles, and F.J. Sanchez-Sesma, "Foundation isolation from vibration using piles as barriers", J. Eng. Mech., ASCE, Vol. 114, pp. 1854-1870, 1988.

[5] S. E. Kattis, D. Polyzos, and D. E. Beskos, "Modelling of pile wave barriers by effective trenches and their screening effectiveness", Soil Dyn. Earthquake Eng., Vol. 18, pp. 1-10, 1999.

[6] S. E. Kattis, D. Polyzos, and D. E. Beskos, "Vibration isolation by a row of piles using a 3-D frequency domain BEM", Int. J. Numer. Methods Eng., Vol. 46, pp. 713-728, 1999.

[7] P. H. Tsai, Y. F. Zheng, and T. L. Jen, "Three-dimensional analysis of the screening effectiveness of hollow pile barriers for foundation-induced vertical vibration", Comput. Geotech., Vol. 38, pp. 489-499, 2007.

[8] M. A. Biot, "Theory of propagation of elastic waves in a fluidsaturated porous solid, I, low frequency range", J. Acoust. Soc. Am., Vol. 28, pp. 168-178, 1956.

[9] M. A. Biot, "Theory of propagation of elastic waves in a fluidsaturated porous solid, II: higher frequency range", J. Acoust. Soc. Am., Vol. 28, pp. 179-191, 1956.

[10] M. A. Biot, "Mechanics of deformation and acoustic propagation in porous media", J. Appl. Phys., Vol. 33, pp. 1482-1498, 1962.

[11] X. Zeng, and R. K. N. D. Rajapakse, "Dynamic axial load transfer from elastic pile to poroelastic medium", J. Eng. Mech., ASCE, 1999, 125: 1048-1055.

[12] J. H. Wang, X. L. Zhou, and J. F. Lu, "Dynamic response of pile groups embedded in a poroelastic medium", Soil Dyn. Earthquake Eng., Vol. 23, pp. 235-242, 2003.

[13] B. Jin, and Z. Zhong, "Lateral dynamic compliance of pile embedded in poroelastic half-space", Soil Dyn. Earthquake Eng, Vol. 21, pp. 519-525, 2001.

[14] J. F. Lu, and D. S. Jeng "Poroelastic model for pile-soil interaction in a half-space porous medium due to seismic waves", Int. $J$. Numer. Anal. Meth. Geomech., Vol. 32, pp. 1-41, 2008.

[15] Y. Q. Cai, G. Y. Ding, and C. J. Xu, "Screening of plane S waves by an array of rigid piles in poroelastic soil", J. Zhejiang Univ. Sci. A, Vol. 9, pp. 589-599, 2008.

[16] Y. Q. Cai, G. Y. Ding, and C. J. Xu, "Amplitude reduction of elastic waves by a row of piles in poroelastic soil", Comput. Geotech, Vol. 36, pp. 463-473, 2009.

[17] J.F. Lu, B. Xu, and J. H. Wang, "Numerical analysis of isolation of the vibration due to moving loads using pile rows", J. Sound Vibrat., Vol. 319, pp. 940-962, 2009.

[18] J.F. Lu, B. Xu, and J. H. Wang, "A numerical model for the isolation of moving-load induced vibrations by pile rows embedded in layered porous media", Int. J. Solids Struct., Vol. 46, pp. 37713781, 2009. 
[19] R. Muki, and E. Sternberg, "On the diffusion of an axial load from an infinite cylindrical pile embedded in an elastic medium", Int. J. Solids Struct., Vol. 5, pp. 587-606, 1969.

[20] R. Muki, and E. Sternberg, "Elastostatic load transfer to a halfspace from a partially embedded axially loaded rod", Int. J. Solids Struc., Vol. 6, pp. 69-90, 1970.

[21] I. N. Sneddon, Ed. Fourier transforms, New York: McGraw-Hill, 1951.

[22] G. Bonnet, "Basic singular solutions for poroelastic medium in the dynamic range", J. Acoust. Soc. Am., Vol. 82, pp. 1758-1762, 1987.

[23] J.F. Lu, D. S. Jeng, and W. D. Nie, "Dynamic response of a pile embedded in a porous medium and subjected to plane SH waves", Comput. Geotech., Vol. 33, pp. 404-418, 2006.
[24] R. Muki, Ed. Asymmetric problem of the theory of elasticity for a semi-infinite solid and a thick plate. In: Progress in Solid Mechanics 1, Interscience, vol. 1, New York, Amaterdam North Holland, 1960.

[25] R.Y. Pak, and P. C. Jennings, "Elasticdynamic response of pile under transverse excitations", J. Eng. Mech., ASCE, Vol. 113, pp 1101-1116, 1987.

[26] R.D. Woods, "Screening of surface waves in soil", J. Soil Mech. Found. Eng., ASCE, Vol. 94(SM4), pp. 951-79, 1968.

[27] B. Jin, "Dynamic displacements of an infinite beam on a poroelastic half-space due to a moving oscillating load", Arch. Appl. Mech., Vol. 74, pp. 277-287, 2004

(C) Xu et al.; Licensee Bentham Open.

This is an open access article licensed under the terms of the Creative Commons Attribution Non-Commercial License (http://creativecommons.org/licenses/by-nc/3.0/) which permits unrestricted, non-commercial use, distribution and reproduction in any medium, provided the work is properly cited. 